\title{
Size and Structure of Dry and Moist Reversible Tropical Cyclones
}

\author{
DANYANG WANG AND YANLUAN LIN \\ Ministry of Education Key Laboratory for Earth System Modeling, Department of Earth System Science, \\ Tsinghua University, Beijing, China
}

(Manuscript received 24 August 2019, in final form 30 March 2020)

\begin{abstract}
The size and structure of tropical cyclones (TCs) are investigated using idealized numerical simulations. Three simulations are conducted: a pure dry TC (DRY), a moist reversible TC (REV) with fallout of hydrometeors in the atmosphere disallowed, and a typical TC (CTL). It was found that the width of the eyewall ascent region and the radius of maximum wind $r_{m}$ are much larger in DRY and REV than those in CTL. This is closely related to the deep inflow layer $(\sim 4 \mathrm{~km})$ in DRY and REV associated with a different entropy restoration mechanism under the subsidence region. With the wide ascents, the close link between $r_{m}$ and the outer radius in DRY and REV can be well predicted by the Emanuel and Rotunno (ER11) model. The magnitude of subsidence, mainly controlled by the vertical gradient of entropy in the mid- and upper troposphere, is nearly one order greater in DRY and REV than that in CTL. This study demonstrates that the falling nature of hydrometeors poses a strong constraint on the size and structure of real world TCs via the entropy distribution in the subsidence region. The wide ascent, self-stratification in the outflow, and decently reproduced wind profile in DRY and REV suggest that DRY and REV behave like a prototype of the ER11 model with CTL being an extreme type.
\end{abstract}

\section{Introduction}

Tropical cyclones (TCs) have been known as warmcore cyclones powered by sea surface enthalpy fluxes (e.g., Emanuel 1986). While theoretical works have demonstrated that its steady-state intensity is bounded by some environmental parameters, such as the air-sea enthalpy disequilibrium, and temperature difference between sea surface and tropopause (Emanuel 1986, 1988, 1991; Bister and Emanuel 1998; Emanuel and Rotunno 2011, hereafter ER11), a theory of its size and structure has not been completely established.

One way to understand the physics of $\mathrm{TC}$ is by comparing a typical TC with an analog, like a dry TC. Mrowiec et al. (2011) demonstrated that an axisymmetric dry TC, in which no moisture and condensation was allowed, can also exist based on Emanuel's theory (e.g., Emanuel 1986). Inspired by Mrowiec et al. (2011), Cronin and Chavas (2019, hereafter CC19) further examined the structure transition from moist to dry TCs. Both studies showed distinct structures between moist and dry TCs: 1) as the TC becomes dry, the ascending region becomes substantially larger compared to the size

Corresponding author: Yanluan Lin, yanluan@tsinghua.edu.cn of TC, with a correspondingly larger radius of maximum wind $r_{m}$ as well; 2) the outer region subsidence velocity is an order of magnitude larger in the dry TC than moist $\mathrm{TC} ; 3)$ the inflow layer of the dry $\mathrm{TC}$ is much deeper than the moist TC; and 4) there is a significant low-level lowentropy layer in the moist TC but not in the dry TC. These distinctions provide valuable opportunities to further understand the physics of TC. Nonetheless, a better understanding of these distinctions requires more extensive and quantitative analyses. On the other hand, how moisture modulates TC size and structure is not yet explicit enough. A primary aim of this study is to further narrow the gap between the dry and moist TC via revealing the role of moisture in constraining TC size and structure. As a hypothesis, it is not moisture itself but the subsaturation of a moist atmosphere due to fallout of hydrometeors that causes the distinctions between typical moist TCs and dry TCs.

To test this hypothesis, an important transition between dry and moist TCs, a so-called moist reversible TC, is explored in this study. A moist reversible TC is one with condensed water following the air parcel rather than falling out of the atmosphere. We expect such a TC to be equivalent to the dry TC for the following reasons. 1 ) The sign of buoyancy does not change with mixing in 
dry or phase equilibrium conditions; specifically, the buoyancy is proportional to the fraction of updraft air in the mixed air parcel. Such an equivalent property is expected to result in similar deep convective activities in dry and moist reversible TCs. In contrast, buoyancy will be reduced and even become negative in a typical moist convection due to condensate evaporation with mixing. 2) With total water mixing ratio nearly conserved, no lower- and midtropospheric low-entropy layer as in a typical moist TC is expected to form. 3) The mechanical efficiency of the moist reversible TC may significantly increase as the atmosphere becomes saturated everywhere and close to that of a dry atmosphere (Pauluis and Held 2002a,b; Pauluis 2011).

As will be demonstrated later, the structures of the moist reversible TC and the dry TC are indeed essentially the same. Thus, moist reversible $\mathrm{TC}$ acts as a natural transition from a dry TC to a typical TC because of its similar thermodynamical and dynamical nature to dry TC with moisture included. The distinction between a moist reversible TC and a typical moist TC is the falling of hydrometeors in the atmosphere. Because of their equivalent nature, we denote dry and moist reversible TCs as dry-type TCs and study their differences from a typical TC in this study.

As will be shown below, the ascent region of dry-type TCs can be several hundred kilometers wide, so the most suitable starting point of understanding the behavior of dry-type TCs may be Emanuel's potential intensity (PI) model (ER11) because it is built fundamentally on the thermodynamic structure of the ascent region. For this reason, the ER11 model is hypothesized to be better realized in dry-type TCs than in a typical moist TC. The ER11 model gives TC structure under the eyewall and the radial wind profile, but it has not been fully validated. For example, the relation between $r_{m}$ and the outer radius is not easy for evaluation in a typical moist TC due to its narrow ascent region. This study thus provides a good opportunity to systematically evaluate and see to what extent size and structure of dry-type TCs can be explained by the ER11 model, following the analyses of $\mathrm{CC} 19$.

In particular, we examine the effect of asymmetry in the ER11 model's boundary layer closure combining entropy and absolute angular momentum budgets under the eyewall (Bryan and Rotunno 2009). ER11 assumed that the outflow temperature is constrained by a critical Richardson number ( $\mathrm{Ri}$ ), indicating a critical state for small-scale turbulence to develop. This assumption is under debate and warrants further investigation. Though the criticality of $\mathrm{Ri}$ in the outflow of an axisymmetric TC is generally found valid (Persing et al. 2013; Peng et al. 2018), its validity in a three-dimensional
TC has not reached a consensus (Persing et al. 2013; Tao et al. 2019; Montgomery et al. 2019). On the other hand, the role of Ri-related turbulent diffusion is found not essential to TC intensification (Montgomery et al. 2019), not supporting the corresponding intensification theory (Emanuel 2012). In this study, we test the critical Ri assumption in more generalized three-dimensional drytype TCs.

Overall, this study aims to

1) demonstrate the equivalent nature of dry and moist reversible TCs;

2) evaluate various aspects of the ER11 model in drytype TCs;

3) explore the dominant factors for the different size and structure between dry-type and typical TCs.

In the following, we first give a brief review of the ER11 model and the entropy budget as well as the TC intensity and size metrics to be used in section 2 . The experimental design of three idealized simulations is described in section 3. Evolution and steady state structures of the simulated TCs are in section 4. Evaluation of the ER11 model and analyses of the underlying mechanisms are given in section 5 followed by the conclusions and discussion in section 6 .

\section{ER11 model and entropy budget}

\section{a. A brief review of the ER11 model}

The ER11 model is based on an axisymmetric framework assuming gradient wind balance, hydrostatic balance, slantwise neutrality, and phase equilibrium (or pure dry). The third assumption also means the entropy is a function of absolute angular momentum alone. It is possible to derive a thermal wind relation from the assumptions [Eq. (12) of ER11]

$$
\frac{v_{b}}{r_{b}}=-\left(T_{b}-T_{o}\right) \frac{d s^{*}}{d M},
$$

where $v_{b}$ is the gradient wind at the top of the boundary layer, $r_{b}$ the radius of $v_{b}, T_{b}$ the constant temperature at the top of boundary layer, $T_{o}$ the outflow temperature where tangential wind is zero on the absolute angular momentum surface of $v_{b}$ in the outflow, $s^{*}$ the saturation entropy (dry entropy in a dry TC), and $M$ the absolute angular momentum. The value of $d s^{*} / d M$ is determined by a boundary layer closure in which $s^{*}$ and $M$ sources are balanced by radial advection [corresponding to Eq. (17) of ER11]:

$$
\frac{d s^{*}}{d M} \approx-\frac{F_{s}}{\tau_{M}}
$$


where $F_{S}$ and $\tau_{M}$ are surface diffusion fluxes of entropy and absolute angular momentum per unit area, respectively. The equations for $F_{s}$ and $\tau_{M}$ are [corresponding to Eqs. (19) and (20) of ER11]

$$
\begin{aligned}
F_{s} & =\frac{C_{k} \rho_{d}|\mathbf{V}|\left(k_{0}^{*}-k\right)}{T_{s}}, \\
\tau_{M} & =C_{D} \rho_{d} r|\mathbf{V}| V,
\end{aligned}
$$

where $C_{k}$ and $C_{D}$ are surface exchange coefficients of enthalpy and momentum, $\rho_{d}$ the density of dry air, $k_{0}^{*}$ the saturation enthalpy at sea surface temperature (SST) (simply the dry enthalpy at SST for dry TC), $k$ the actual enthalpy of near surface air, $T_{s}$ the surface air temperature, $|\mathbf{V}|$ the surface total horizontal wind, $V$ the surface tangential wind, and $r$ the radius. It is then generally assumed that $V \approx v_{b}$, and $r \approx r_{b}$. Note that $T_{o}$ is a function of $M$ by relating $\partial T_{o} / \partial M$ to $d M / d s^{*}$ [Eq. (31) of ER11]:

$$
\frac{\partial T_{o}}{\partial M} \cong-\frac{\mathrm{Ri}}{r_{t}^{2}}\left(\frac{d M}{d s^{*}}\right)
$$

where $r_{t}$ is the constant radius corresponding to Richardson number $\mathrm{Ri}$ in the outflow. As $d M / d s^{*}$ is negative [Eq. (2)], $\partial T_{o} / \partial M$ would be positive, so that $T_{o}$ increases with $M$.

With $M=r v+(1 / 2) f r^{2}$, where $f$ is the Coriolis parameter, the system is complete with proper boundary conditions to solve $s^{*}$ and $T_{o}$ as a function of $M$ as well as the corresponding $v_{b}$ (ER11). If $f r \ll v$ is assumed, it is also possible to derive an analytical solution of the system (ER11).

\section{b. Entropy budget}

Entropy $s$ is defined following Pauluis (2011) as

$$
s=\left(c_{p}+q_{t} c_{l}\right) \ln \frac{T}{T_{0}}-R_{d} \ln \frac{p_{d}}{p_{0}}+\frac{L_{v} q_{v}}{T}-q_{v} R_{v} \ln \mathscr{H},
$$

where $c_{p}$ is the specific heat of dry air at constant pressure, $q_{t}$ the total water mixing ratio, $c_{l}$ the specific heat of liquid water at constant volume, $T$ the temperature, $T_{0}$ an arbitrary reference temperature set to $273.15 \mathrm{~K}, R_{d}$ the gas constant of dry air, $p_{0}$ an arbitrary reference pressure set to $1000 \mathrm{hPa}, L_{v}$ the latent heat of vaporization, $q_{v}$ the water vapor mixing ratio, $R_{v}$ the gas constant of water vapor, and $\mathscr{H}$ the relative humidity.

An entropy budget consistent with Pauluis and Held (2002a,b) and Romps (2008) may be given as

$$
\frac{\partial s}{\partial t}=\mathrm{ADV}_{h}+\mathrm{ADV}_{v}+\delta S_{\mathrm{rad}}+\delta S_{\mathrm{sflx}}+\delta S_{\mathrm{wflx}}+\delta S_{\mathrm{irr}},
$$

where $\partial s / \partial t$ is the local tendency of entropy, the first two terms on the RHS are respectively the horizontal and vertical advection of entropy, $\delta S_{\text {rad }}$ the entropy sink from radiative cooling, $\delta S_{\text {sflx }}$ the entropy source from convergence of sensible heat diffusion fluxes, $\delta S_{\mathrm{wflx}}$ the entropy source from convergence of water vapor and liquid water diffusion fluxes, and $\delta S_{\text {irr }}$ the sum of irreversible entropy sources from viscous dissipation, dissipative heating of falling hydrometeors, diffusion of water vapor, and irreversible phase changes in the atmosphere. The detailed definitions of entropy source/sink terms are referred to Pauluis and Held (2002a,b) and Romps (2008). All terms of Eq. (7) are azimuthally averaged for presentation in section 5 .

\section{c. Intensity and size metrics}

We measure intensity mainly by the maximum tangential wind $v_{m}$ at $1 \mathrm{~km}$ of altitude, approximately at the top of the boundary layer, a height corresponding to the derivation in ER11. Size is measured by the radius of $v_{m}\left(r_{m}\right), 17 \mathrm{~m} \mathrm{~s}^{-1}\left(r_{17}\right)$, and $4 \mathrm{~m} \mathrm{~s}^{-1}\left(r_{4}\right)$ tangential wind at $1 \mathrm{~km}$ of altitude. Considering different widths of the secondary circulation between dry-type and typical moist TCs, different from traditional size metrics, a mass streamfunction describing mass fluxes in radius-height section, is also used to describe the size of TCs (see section 4).

\section{Experimental design}

The model used in this study is Cloud Model 1 (CM1), release 19.2 (Bryan and Fritsch 2002), a threedimensional, nonhydrostatic numerical model mainly designed for idealized simulations. All simulations used the same model configuration as described below. The domain size is $3000 \mathrm{~km} \times 3000 \mathrm{~km}$ in the horizontal and $25 \mathrm{~km}$ in the vertical. The horizontal grid spacing is $3 \mathrm{~km}$ in the inner $600 \mathrm{~km} \times 600 \mathrm{~km}$ area and stretches to $12 \mathrm{~km}$ at the outer edge. The vertical grid spacing is $200 \mathrm{~m}$ in the lowest $2 \mathrm{~km}$ of altitude and stretches to $1 \mathrm{~km}$ above the altitude of $17 \mathrm{~km}$. The subgrid turbulences for vectors and scalars are parameterized for mesoscale modeling $(\mathrm{cm} 1$ setup $=2, \mathrm{ipbl}=2$ in the namelist $)$ with the horizontal and asymptotic vertical mixing lengths $\left(l_{h}\right.$ and $\left.l_{\infty}\right)$ set to $1000 \mathrm{~m}($ lhref1 $=1 \mathrm{href} 2=1000)$ and $50 \mathrm{~m}(1$ inf $=50)$, respectively. The values are comparable to those used in Montgomery et al. (2019). Surface exchange coefficients for momentum $c_{D}$ and enthalpy $c_{k}$ are 0.002 and 0.0015 , respectively. Note that a critical 
TABLE 1. Summary of the three experiments.

\begin{tabular}{lllr}
\hline \hline Expt & \multicolumn{1}{c}{ Base-state sounding } & Microphysics & $\begin{array}{c}\text { Air-sea temperature } \\
\text { difference (K) }\end{array}$ \\
\hline DRY & Dry adiabatic, no water & - & 13 \\
REV & Moist adiabatic, total water conserved & Warm rain, no fallout of hydrometeors in the atmosphere & 5 \\
CTL & Pseudoadiabatic & Warm rain & 5 \\
\hline
\end{tabular}

$\mathrm{Ri}$ of 1 exists via the calculation of the vertical viscosity $K_{m, v}$ :

$$
K_{m, v}=l_{v}^{2} S_{v}(1-\mathrm{Ri} / \mathrm{Pr})^{1 / 2},
$$

where Pr is the Prandtl number set to $1, l_{v}$ the vertical mixing length, and $S_{v}^{2}=\left(\partial u_{x} / \partial z+\partial w / \partial x\right)^{2}+\left(\partial u_{y} / \partial z+\right.$ $\partial w / \partial y)^{2}$ the deformation with $u_{x}, u_{y}$, and $w$ the velocities in Cartesian coordinates. $\mathrm{Ri}$ is defined as

$$
\mathrm{Ri}=\frac{N_{m}^{2}}{S_{v}^{2}}
$$

with $N_{m}$ the buoyancy frequency. We set $K_{m, v}$ to zero when $\mathrm{Ri}>1$. For simplicity, the radiation is represented by a Newtonian cooling capped at $2 \mathrm{~K} \mathrm{day}^{-1}$ (Rotunno and Emanuel 1987). Dissipative heating is also turned on for the conservation of energy. The specification of the initial vortex follows Rotunno and Emanuel (1987). The sea surface temperature is set to $301.15 \mathrm{~K}$. All simulations are integrated for 30 days.

Three experiments are designed and summarized in Table 1. The first experiment is a dry TC (DRY), designed following Mrowiec et al. (2011). The initial sounding is dry adiabatic from the surface to $10 \mathrm{~km}$ representing the troposphere with a similar temperature difference $(\sim 100 \mathrm{~K})$ between the surface and tropopause to that over the tropics (Fig. 1a). Above $10 \mathrm{~km}$ of altitude the potential temperature increases exponentially with height to represent the stratosphere (Fig. 1a). To give enough surface enthalpy flux to power the dry TC, initial surface air temperature is set $13 \mathrm{~K}$ cooler than SST. Note that this value is about the lowest for a dry TC to develop in the present model configuration. In DRY, there is no mass of water in the initial sounding (Fig. 1b) and the surface moisture flux is also turned off during the simulation.

The second experiment is a moist reversible TC (REV). The initial sounding is a moist reversible adiabatic profile conserving moist entropy (Emanuel 1994) from the surface to $15 \mathrm{~km}$ of altitude, representing the troposphere (Fig. 1a). The surface air is saturated and the total water mixing ratio is kept constant up to $15 \mathrm{~km}$ of altitude, with the condensed liquid water left in the air (Fig. 1b). Above $15 \mathrm{~km}$ of altitude, the virtual potential temperature $\theta_{v}$ increases exponentially with height, while also conserving the total water mixing ratio. The initial surface air temperature is set $5 \mathrm{~K}$ cooler than SST to support a moist reversible TC. This temperature difference is about the lowest for a TC to develop in REV, though it exceeds that necessary for a typical moist TC in nature (e.g., Črnivec et al. 2015). In REV, the microphysics scheme is a warm-rain scheme (Kessler 1995), but autoconversion of cloud to rain is turned off so that the moist thermodynamic transformations are nearly reversible as fallout of rain is eliminated. However, to balance infinite net water mass input to the atmosphere due to nonnegligible moisture flux, an artificial "diffusion" of liquid water to the sea is added to ensure conservation of total water. It was accomplished by the removal of extra liquid water when liquid water mixing ratio was above $0.001 \mathrm{~kg} \mathrm{~kg}^{-1}$ at the lowest model level.

The third experiment is a typical moist TC (CTL) with an equivalent initial environment of REV. The purpose of this experiment is to exclude the influence of the large air-sea temperature difference on TC structure and explicitly show the impact of the falling nature of hydrometeors in the atmosphere. The initial sounding is pseudoadiabatic (Bolton 1980; Emanuel 1994) from the surface to $15 \mathrm{~km}$ of altitude, representing the troposphere, with surface air just saturated (Figs. 1a,b). The sounding is a bit colder in CTL than REV because of conserving a pseudoadiabatically defined entropy with the neglect of heat capacity of condensed water (Emanuel 1994). The air-sea temperature difference is set to $5 \mathrm{~K}$, which is the same as REV. In CTL, the microphysics scheme is the same as REV, but allowing autoconversion of cloud to rain and allowing rain to fall at the default terminal fall speed.

\section{Evolution and structure of simulated TCs}

The temporal evolution of intensity and structure in terms of central surface pressure, $v_{m}, r_{m}, r_{17}$, and $r_{4}$ (Fig. 2) displays significant contrasts among the three simulations. The maximum intensity and intensification rate are much larger in CTL than those in dry-type TCs. The radius $r_{m}$ reaches $\sim 220 \mathrm{~km}$ in both DRY and REV, but it essentially remains within $30 \mathrm{~km}$ in CTL. Similarly, $r_{17}$ reaches $\sim 560 \mathrm{~km}$ in DRY and $\sim 620 \mathrm{~km}$ in REV, but it is about $200 \mathrm{~km}$ in CTL. Differences also hold in 
(a) $\mathrm{T}$

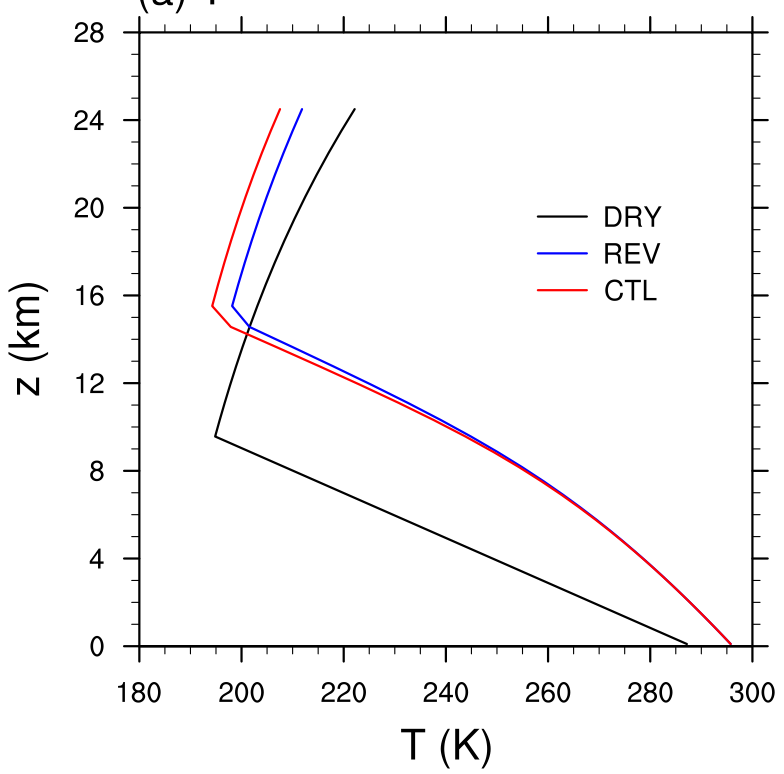

(b) qt

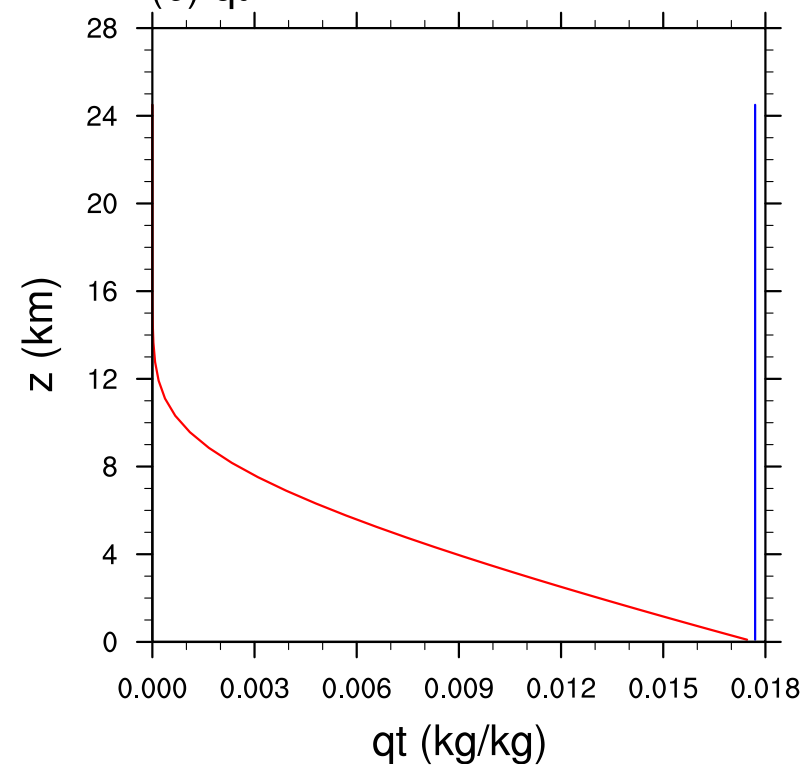

FIG. 1. Base-state sounding in the three experiments: DRY (black), REV (blue), and CTL (red) showing (a) temperature $(\mathrm{K})$ and (b) total water mixing ratio $\left(\mathrm{kg} \mathrm{kg}^{-1}\right)$.

$r_{4}: \sim 800 \mathrm{~km}$ in DRY, $\sim 900 \mathrm{~km}$ in REV, and $\sim 650 \mathrm{~km}$ in CTL. The wide wind fields in DRY and REV also result in much larger domain-integrated kinetic energy than CTL (Fig. 2f). In the following analyses, we mainly focused on the structure during the steady state. The period of $600-700 \mathrm{~h}$ is chosen for all experiments, mainly based on the steadiness of size and intensity as shown in Fig. 2.

The structures of secondary circulation and thermodynamics are illustrated by the radius-height sections of

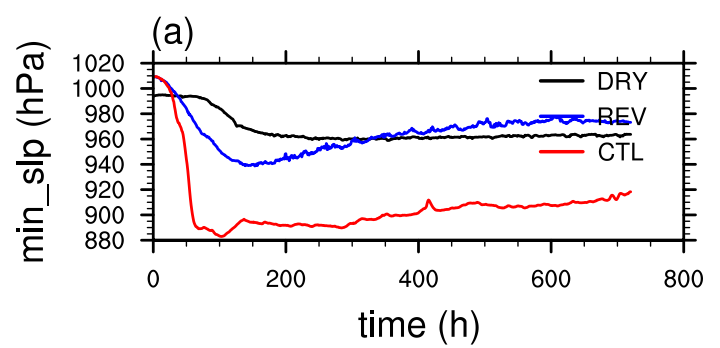

(b)
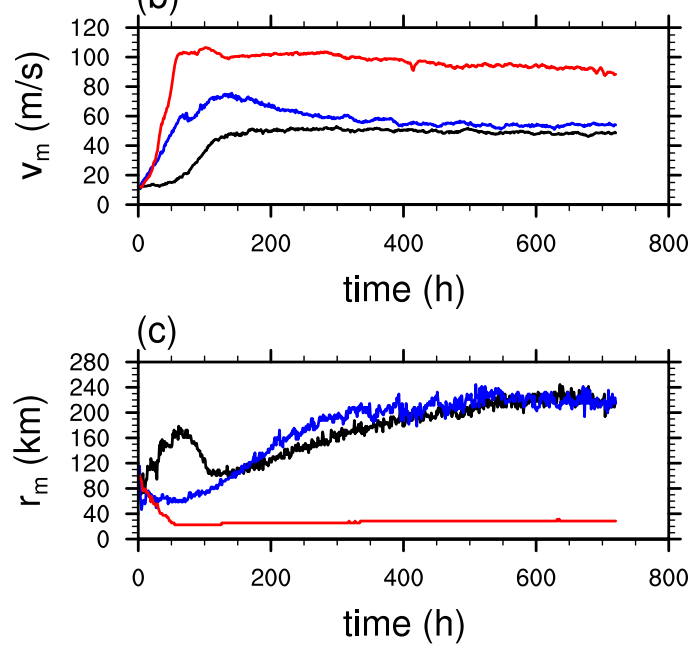

(d)

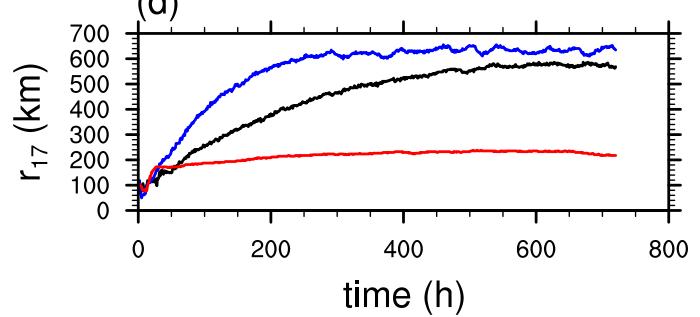

(e)
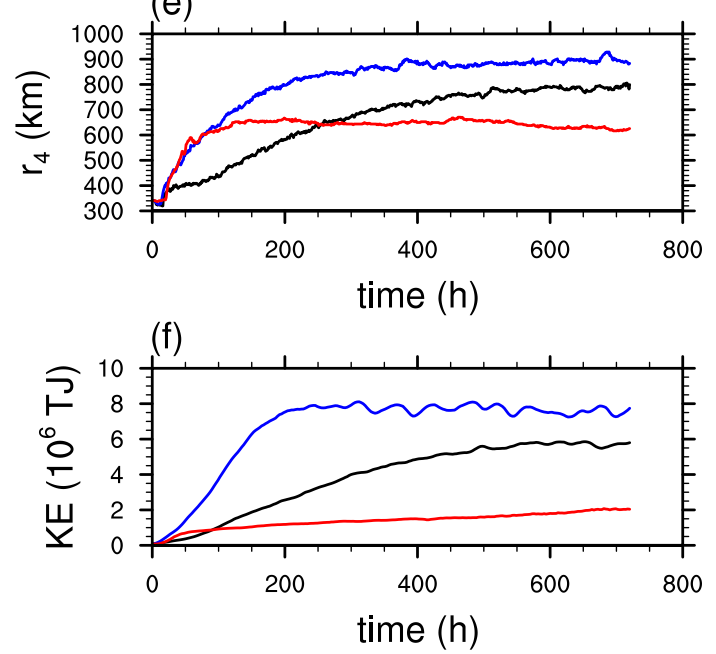

FIG. 2. Temporal evolution of (a) the central surface pressure (hPa), (b) maximum tangential wind $v_{m}\left(\mathrm{~m} \mathrm{~s}^{-1}\right)$ at $1 \mathrm{~km}$ of altitude, (c) radius of $v_{m}\left(r_{m} ; \mathrm{km}\right),(\mathrm{d})$ radius of the $17 \mathrm{~m} \mathrm{~s}^{-1}$ tangential wind at $1 \mathrm{~km}$ of altitude $\left(r_{17} ; \mathrm{km}\right)$, (e) radius of the $4 \mathrm{~m} \mathrm{~s}^{-1}$ tangential wind at $1 \mathrm{~km}$ of altitude $\left(r_{4} ; \mathrm{km}\right)$, and (f) domain-integrated kinetic energy $\left(10^{6} \mathrm{TJ}\right)$ of the three experiments: DRY (black), REV (blue), and CTL (red). 
mass streamfunction and entropy in Fig. 3. The mass streamfunction $\psi$ is defined as

$$
\frac{\partial \psi}{\partial r}=\bar{\rho} r \bar{w},
$$

where $\rho$ is air density, and the overbar denotes an azimuthal average.

To give a quantitative measure of the secondary circulation structure, we define some parameters: $\psi_{\max }$ the value of maximum $\psi$, whose radius is $r_{\psi_{\max }}, r_{\psi_{0}}$ the radius where $\psi$ is zero at a certain altitude, and $r_{\psi_{0}} / r_{\psi_{\max }}$ a ratio reflecting the relative radial width of ascent (including the eye) to the total width of secondary circulation. For DRY and REV, the altitude of evaluation is just the altitude of $\psi_{\max }$, while for CTL, we arbitrarily set the altitude to be $5 \mathrm{~km}$, as the altitude of the actual $\psi_{\max }$ $(\sim 13 \mathrm{~km})$ does not represent the ascent structure below $11 \mathrm{~km}$, which is rather compact (Fig. 3c).

One remarkable feature of the secondary circulation in DRY is the comparable width of ascent and descent regions (Fig. 3a). The maximum streamfunction reaches $17 \times 10^{9} \mathrm{~kg} \mathrm{~s}^{-1}$ during the steady state, with the radial size $\sim 2.3$ times of the ascent region (Table 2). The inflow layer is $\sim 4 \mathrm{~km}$ deep. Note that the main region of entropy sink is in the outflow layer; in the descending region, the entropy varies little, consistent with the basestate sounding. The overall structure of REV (Fig. 3b) is similar to DRY. The maximum streamfunction reaches $\sim 17 \times 10^{9} \mathrm{~kg} \mathrm{~s}^{-1}$, with the radial size $\sim 2.2$ times of the ascent region (Table 2).

In contrast, the overall structure of CTL (Fig. 3c) is different from DRY and REV. First, the ascent region is much smaller compared to the descent region in CTL: $r_{\psi_{0}} / r_{\psi_{\max }}=14.9$, about 6-7 times of DRY and REV (Table 2). The width of the ascending region in CTL is much smaller than that in DRY and REV, indicated by an order of magnitude different $r_{\psi_{\max }}$ (Table 2). The maxima of streamfunction are $\sim 12$ times smaller than DRY and REV (Table 2). The much stronger secondary circulation in DRY and REV may correspond to the much larger mechanical work required to maintain their large wind fields with dissipation. Second, different from DRY and REV, there exists strong vertical entropy gradient in the descending region, as found by Mrowiec et al. (2011). Third, the inflow layer is only $\sim 1 \mathrm{~km}$ deep in CTL (Fig. 3).

The percentage of surface frictional dissipation to the total energy input (e.g., CC19) during the steady state is $6.9 \%, 6.0 \%$, and $1.9 \%$ for DRY, REV and CTL, respectively. This suggests that the mechanical efficiency of dry-type TCs may be significantly larger than CTL. Note that a more complete calculation of actual mechanical efficiency is needed in the future.

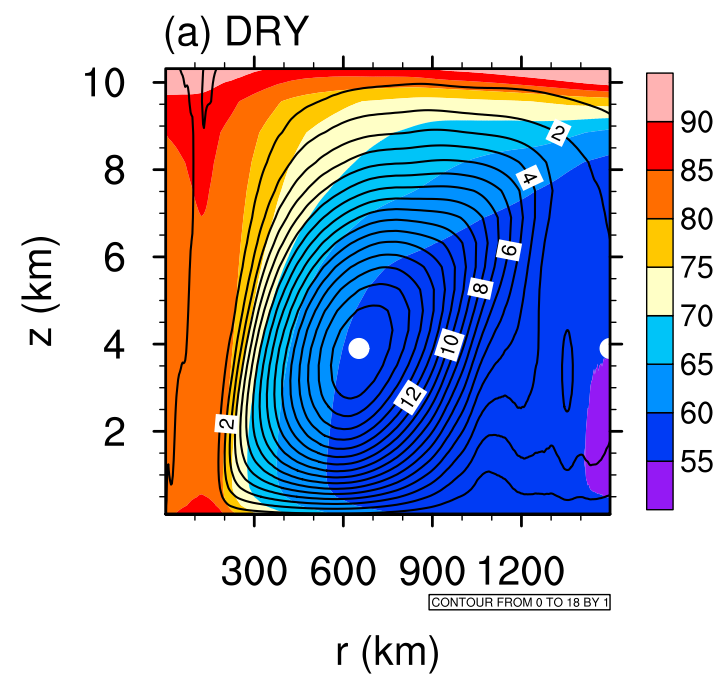

(b) REV

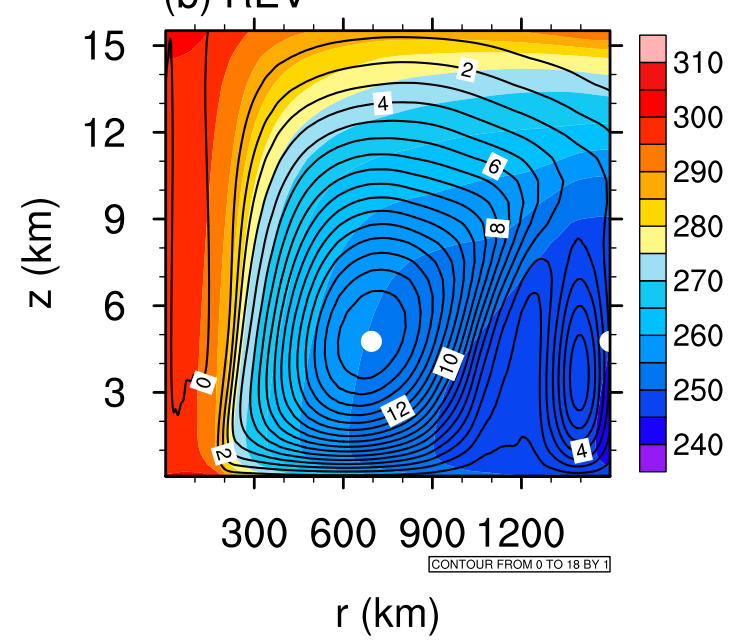

(c) CTL

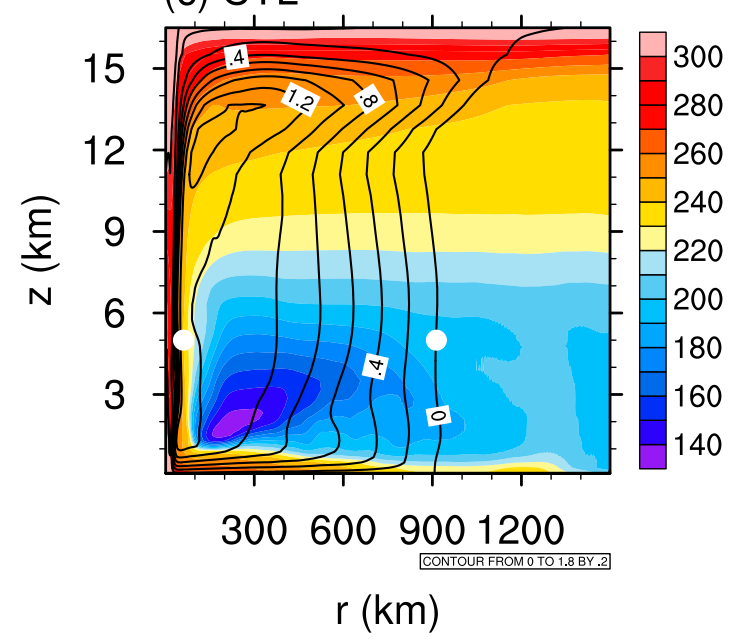

FIG. 3. Height-radius sections of azimuthal mean entropy ( $\mathrm{J} \mathrm{K}^{-1} \mathrm{~kg}^{-1}$; shaded) and mass streamfunction $\left[10^{9} \mathrm{~kg} \mathrm{~s}^{-1}\right.$; contours, with an interval of $1.0 \times 10^{9} \mathrm{~kg} \mathrm{~s}^{-1}$ in (a) and (b) and $0.2 \times$ $10^{9} \mathrm{~kg} \mathrm{~s}^{-1}$ in (c), negative values not shown] of (a) DRY, (b) REV, and (c) CTL during the steady state. The points $\left(r_{\psi_{\max }}, z_{\psi_{\max }}\right)$ and $\left(r_{\psi_{0}}, z_{\psi_{\max }}\right)$ are marked by white-filled circles for each experiment. 
TABLE 2. Statistics of mass streamfunction in the three experiments during the steady state.

\begin{tabular}{lcccc}
\hline \hline Expt & $\psi_{\max }\left(10^{9} \mathrm{~kg} \mathrm{~s}^{-1}\right)$ & $r_{\psi_{\max }}(\mathrm{km})$ & $r_{\psi_{0}}(\mathrm{~km})$ & $r_{\psi_{0}} / r_{\psi_{\max }}$ \\
\hline DRY & 17.0 & 652.5 & 1500.0 & 2.3 \\
REV & 17.0 & 694.5 & 1500.0 & 2.2 \\
CTL & 1.3 & 61.5 & 913.5 & 14.9 \\
\hline
\end{tabular}

\section{Interpretation and discussion of the underlying mechanisms}

\section{a. Evaluation of ER11 model in DRY and REV}

We evaluate the basic assumptions of the ER11 model in this section. The approximate gradient wind balance above the boundary layer $(\sim 1 \mathrm{~km})$ is generally supported in Figs. 4a-i, with the agradient wind generally smaller than $2.5 \mathrm{~m} \mathrm{~s}^{-1}$. The self-stratification of entropy in the outflow is supported by Figs. 3a-c, because the entropy stratification in the outflow does not match that in the ambient environment where entropy is constant in the troposphere. The outflow temperature as a function of $M$ is supported by Figs. $4 \mathrm{j}-1$. Note that self-stratification in the outflow of a typical TC is more carefully demonstrated in ER11. The slantwise neutrality is also supported by Figs. $4 \mathrm{~m}-\mathrm{o}$, though we do see some decrease of $s$ along $M$ surfaces in DRY.

First, we evaluate the boundary layer closure in ER11. For the experiments conducted, a more precise form of Eq. (2) is

$$
\frac{d \overline{s^{*}}}{\overline{d \bar{M}} \approx \frac{\overline{\frac{1}{\rho_{d}} F_{s}}+\int_{0}^{h} \bar{D} \bar{T} d z-\int_{0}^{h} \bar{w} \frac{\partial \bar{s}}{\partial z} d z-\int_{0}^{h}\left(\frac{1}{r} \frac{\partial r \overline{u^{\prime} s^{\prime}}}{\partial r}+\frac{\partial \overline{w^{\prime} s^{\prime}}}{\partial z}\right) d z}{-\frac{1}{\rho_{d}} \tau_{M}}-\int_{0}^{h} \bar{w} \frac{\partial \bar{M}}{\partial z} d z-\int_{0}^{h}\left(\frac{1}{r} \frac{\partial r \overline{u^{\prime} M^{\prime}}}{\partial r}+\frac{\partial \overline{w^{\prime} M^{\prime}}}{\partial z}\right) d z},
$$

where $u$ is the radial velocity, and $h$ the height of the boundary layer top. The overbar indicates azimuthal average and the prime indicates deviation from the azimuthal average. The last two terms of numerator and denominator are the vertical mean advection and convergence of resolved eddy fluxes of entropy and absolute angular momentum, respectively. The approximate equality sign reflects a neglect of the vertical variation of azimuthal mean radial wind and density and the taking dissipative heating as the only irreversible entropy production. If the vertical mean advection and eddy fluxes of entropy and absolute angular momentum are neglected, Eq. (11) would be equivalent to Eq. (17) of Bister and Emanuel (1998).

The diagnosed $d s^{*} / d \bar{M}$ by Eq. (11) without and with the vertical mean advection and convergence of resolved eddy fluxes terms in DRY and REV during the steady state are shown in Figs. 5a and 5b, compared with

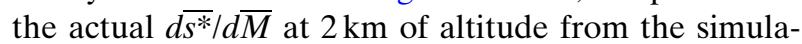
tions. The value of $h$ is set to $1 \mathrm{~km}$ in the calculation of Eq. (11) because it is about the height where subgrid turbulence becomes unimportant (not shown). The diagnosed $d \overline{s^{*}} / d \bar{M}$ with the advection and eddy flux terms shares a similar shape as the actual $d \overline{s^{*}} / d \bar{M}$, especially in the inner edge of the eyewall in DRY and REV, while the diagnosed $d \overline{s^{*}} / d \bar{M}$ without the advection and eddy flux terms also agrees with the actual values elsewhere. The vertical mean advection term in the $M$ budget, smaller than the convergence of resolved eddy fluxes, is over 3 times smaller than the surface flux term in DRY and REV (Figs. 5d,e). In the $s$ budget, the vertical mean advection term appears to be larger than the dissipative heating term near $r_{m}$ (Figs. $5 \mathrm{~g}, \mathrm{~h}$ ). The convergence of resolved eddy fluxes is not much smaller than surface entropy flux term in DRY and REV, especially outside the inner edge of the eyewall (Figs. 5e,f). However, convergence of resolved eddy fluxes seems to compensate for the vertical mean advection to some extent near the inner edge of the eyewall.

In CTL (Fig. 5c), the diagnosed $d s^{*} / d \bar{M}$ is comparable to the actual value around $35 \mathrm{~km}$, but deviates significantly outward to the subsidence region and inward to the eyewall region. Details (Figs. 5f,i) show that contributions of vertical mean advection of both $M$ and $s$ are very strong, but with convergence of resolved eddy fluxes of similar magnitude as DRY and REV.

Another important element in ER11 is the variation of $T_{o}$ as a function of $M$. ER11 found the outflow is controlled by a critical Richardson number Ri of 1 in an axisymmetric simulation. To compare with ER11, Ri is recalculated from the time and azimuthal mean fields. The horizontal gradients of $w$ in the denominator in Eq. (9) are neglected because the denominator is nearly identical to the square of vertical shear of horizontal total wind. Ri during the steady state of the three experiments is generally greater than 1 at the inception of the outflow, where the flow becomes approximately horizontal (indicated by the gray box in Fig. 6), with Ri 
(a) vg

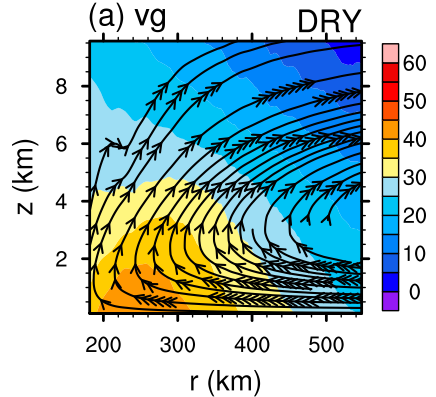

(d) $\mathrm{v}$

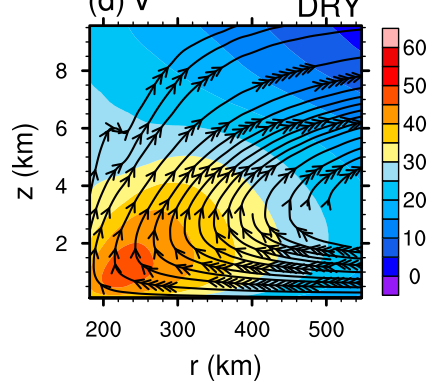

(g) $v$-vg

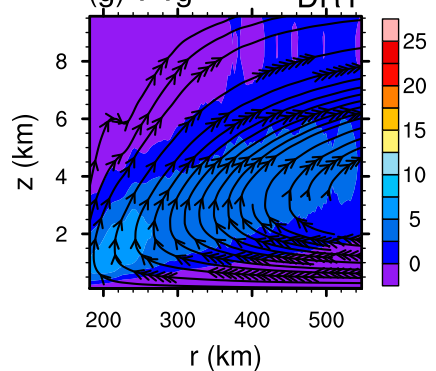

(j) $\mathrm{T}$

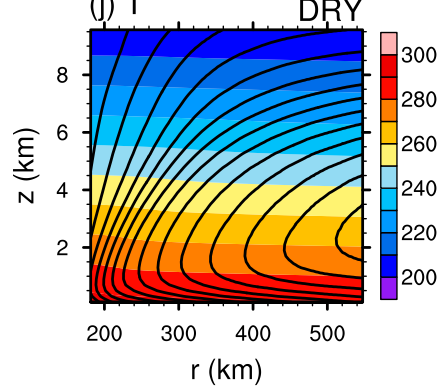

(m) $\mathrm{s}$

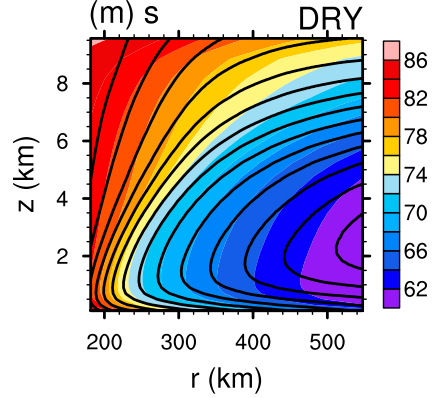

(b) vg

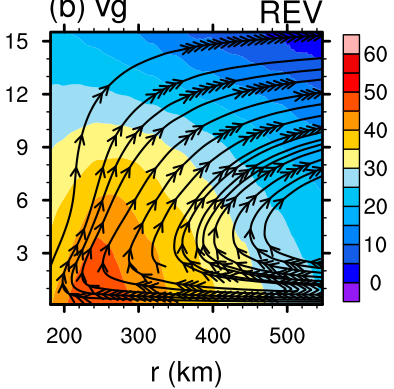

(e) $v$

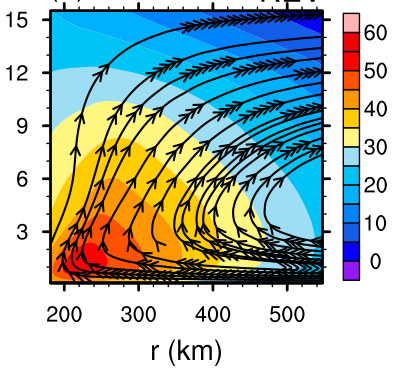

(h) $v-v g$

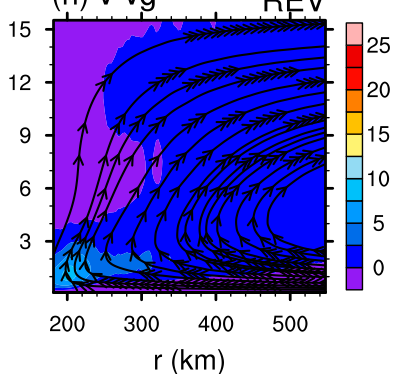

(k) $\mathrm{T}$

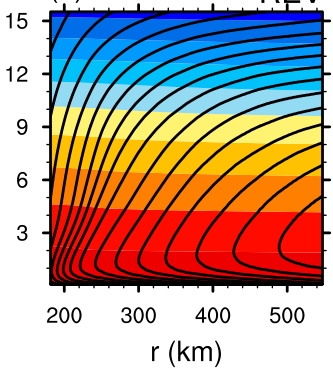

(n) $\mathrm{s}$

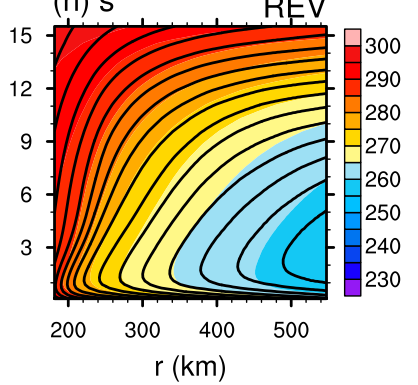

(c) $\mathrm{vg}$

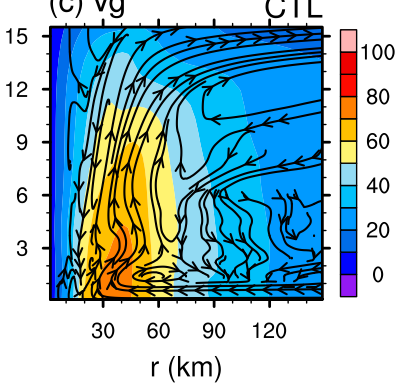

CTL

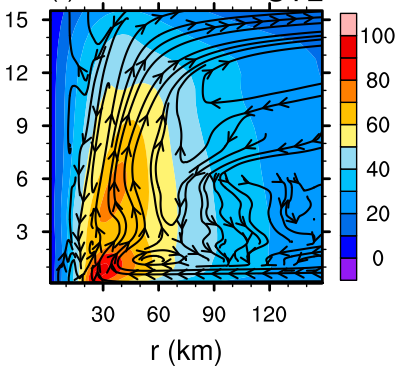

(i) $v-v g$

CTL

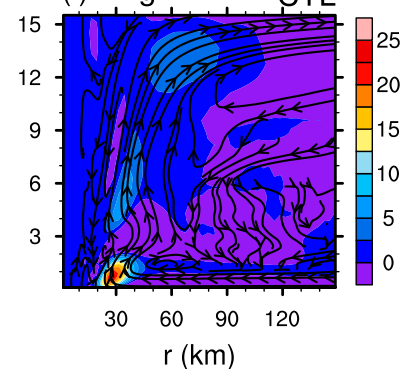

(I) $T$

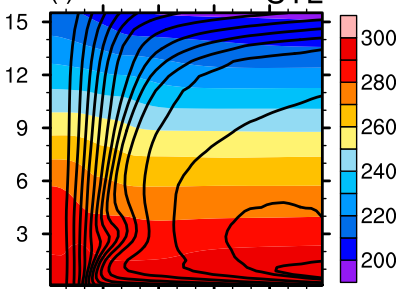

$\begin{array}{llll}30 & 60 & 90 & 120\end{array}$

$\mathrm{r}(\mathrm{km})$

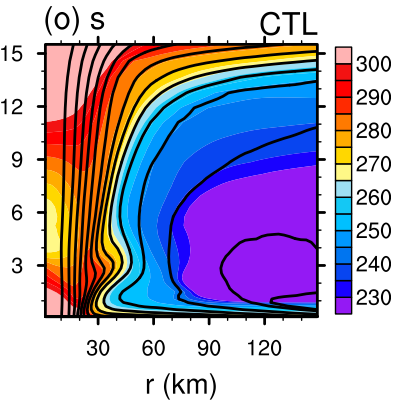

FIG. 4. Azimuthal-mean structures in the ascent region in (left) DRY, (center) REV, and (right) CTL during the steady state: (a)-(c) the gradient wind $\left(\mathrm{m} \mathrm{s}^{-1}\right.$; shaded) overlaid by streamlines, (d)-(f) actual tangential wind $\left(\mathrm{m} \mathrm{s}^{-1}\right.$; shaded) overlaid by streamlines, (g)-(i) agradient wind $\left(\mathrm{m} \mathrm{s}^{-1}\right.$; shaded) overlaid by streamlines, (j)-(1) temperature (K; shaded) overlaid by $M$ (contours, with an interval of $10^{6} \mathrm{~m}^{2} \mathrm{~s}^{-1}$ for DRY and REV and $2 \times 10^{5} \mathrm{~m}^{2} \mathrm{~s}^{-1}$ for CTL), and (m)-(o) entropy $\left(\mathrm{J} \mathrm{K}^{-1} \mathrm{~kg}^{-1}\right.$; shaded) overlaid by $M$ [contours, contour intervals as in (j)-(l)]. 

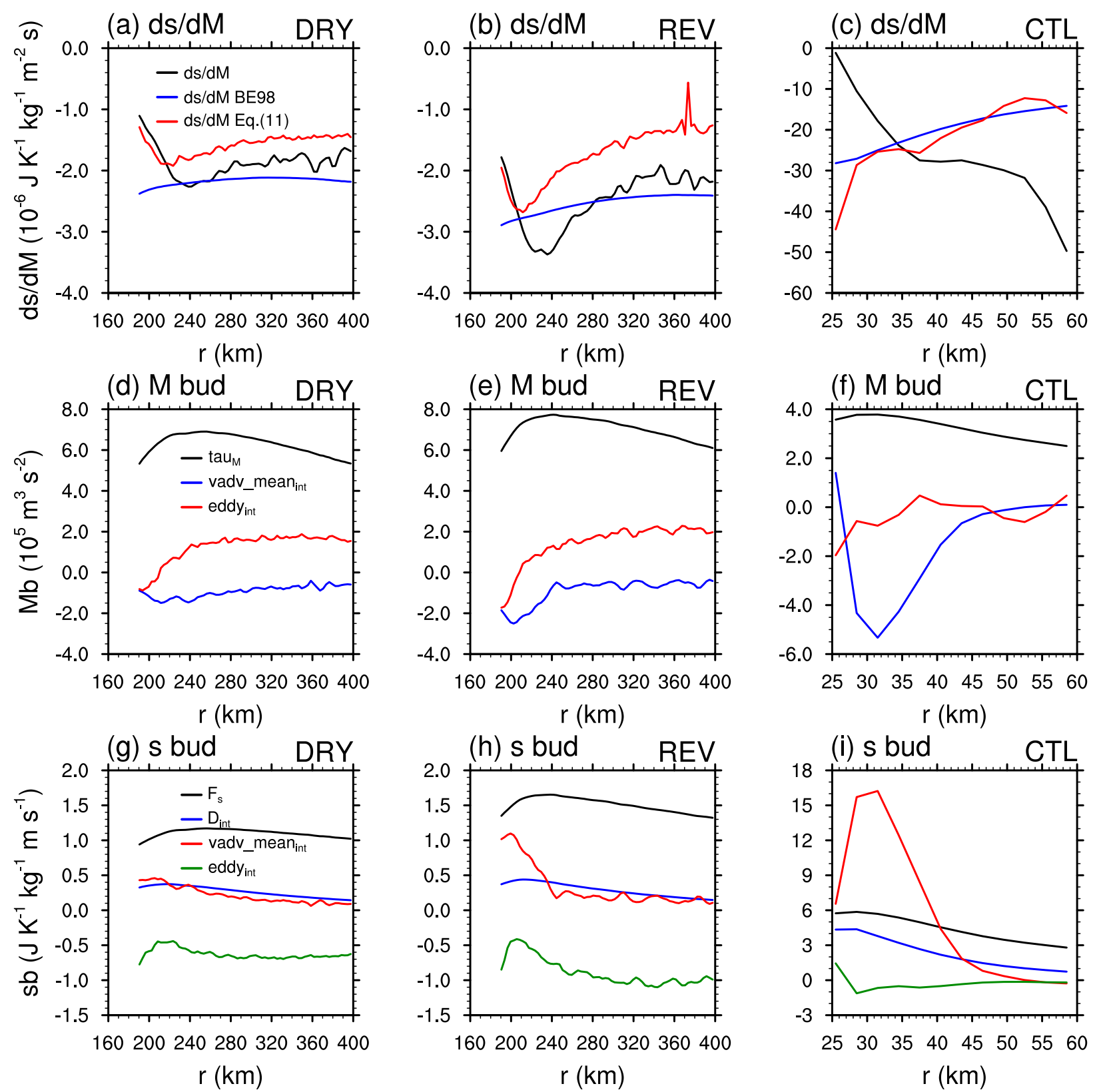

FIG. 5. Actual and diagnosed $d \overline{s^{*}} / d \bar{M}\left(10^{-6} \mathrm{~J} \mathrm{~K}^{-1} \mathrm{~kg}^{-1} \mathrm{~m}^{-2} \mathrm{~s}\right)$ at the top of boundary layer and the corresponding budgets of $M$ $\left(10^{5} \mathrm{~m}^{3} \mathrm{~s}^{-2}\right)$ and $s\left(\mathrm{~J} \mathrm{~K}^{-1} \mathrm{~kg}^{-1} \mathrm{~m} \mathrm{~s}^{-1}\right)$ during the steady state of (left) DRY, (center) REV, and (right) CTL. (a)-(c) The 1-2-1 filtered actual $d \overline{s^{*}} / d \bar{M}$ at $2 \mathrm{~km}$ of altitude (black) and that diagnosed by Eq. (11) without (blue) and with (red) the advection and eddy terms. (d)-(f) The $M$ budget: $\overline{1 / \rho_{d}\left(\tau_{M}\right)}$ term (black), $-\int_{0}^{h} \bar{w} \partial \bar{M} / \partial z d z$ term (blue), and $-\int_{0}^{h}\left[1 / r\left(\partial r \overline{u^{\prime} M^{\prime}} / \partial r\right)+\partial \overline{w^{\prime} M^{\prime}} / \partial z\right] d z$ term (red) in Eq. (11). (g)-(i) The $s$ budget: $\overline{1 / \rho_{d}\left(F_{s}\right)}$ term (black), $\int_{0}^{h} \bar{D} / T d z$ term (blue), $-\int_{0}^{h} \bar{w} \partial \bar{s} / \partial z d z$ term (red), and $-\int_{0}^{h}\left[1 / r\left(\partial r \overline{u^{\prime} s^{\prime}} / \partial r\right)+\partial \overline{w^{\prime} s^{\prime}} / \partial z\right] d z$ term (green) in Eq. (11).

increasing outward (Figs. 6a-c). As instantaneous Ri [Eq. (9)] at each grid point determines the onset of vertical turbulence in CM1, the corresponding fraction of number of grid points with $0<\mathrm{Ri}<1$ along an azimuth is also calculated. The percentage of grid points with $0<\mathrm{Ri}<1$ in the gray box is around $50 \%$ in DRY, $30 \%$ in REV and $20 \%$ in CTL (Figs. 6d-f). This suggests that turbulence is active (in pulses) in the outflow, supporting ER11, though the temporal and azimuthal mean flow fields seem to be subcritical. Nevertheless, with a given Richardson number, it is still possible to obtain Eq. (5) in all simulations.

The dependence of $\partial \overline{T_{o}} / \partial \bar{M}$ on $d \bar{M} / d \overline{s^{*}}$ in Eq. (5) is evaluated in Fig. 7. In each experiment, we focus on a 


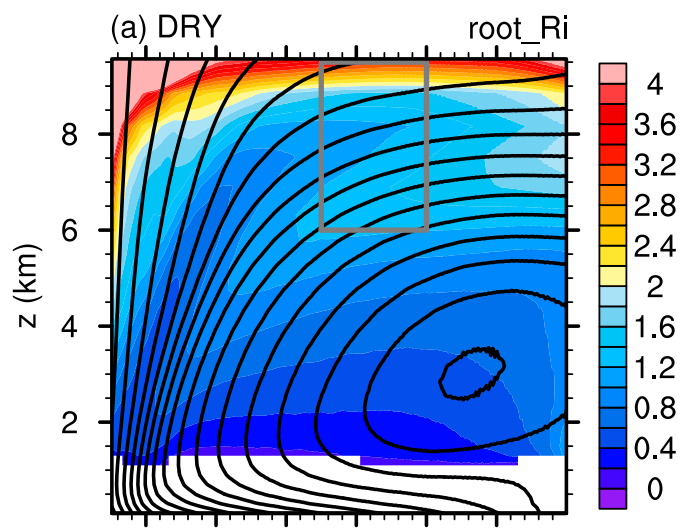

200300400500600700

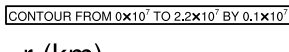

$r(\mathrm{~km})$

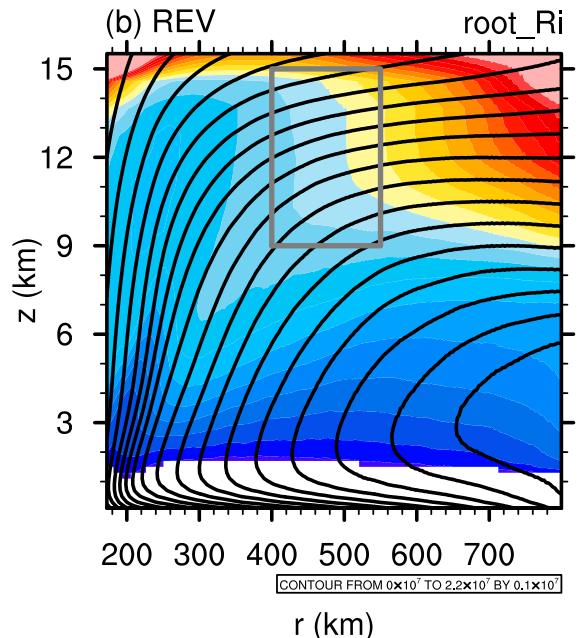

(c) CTL
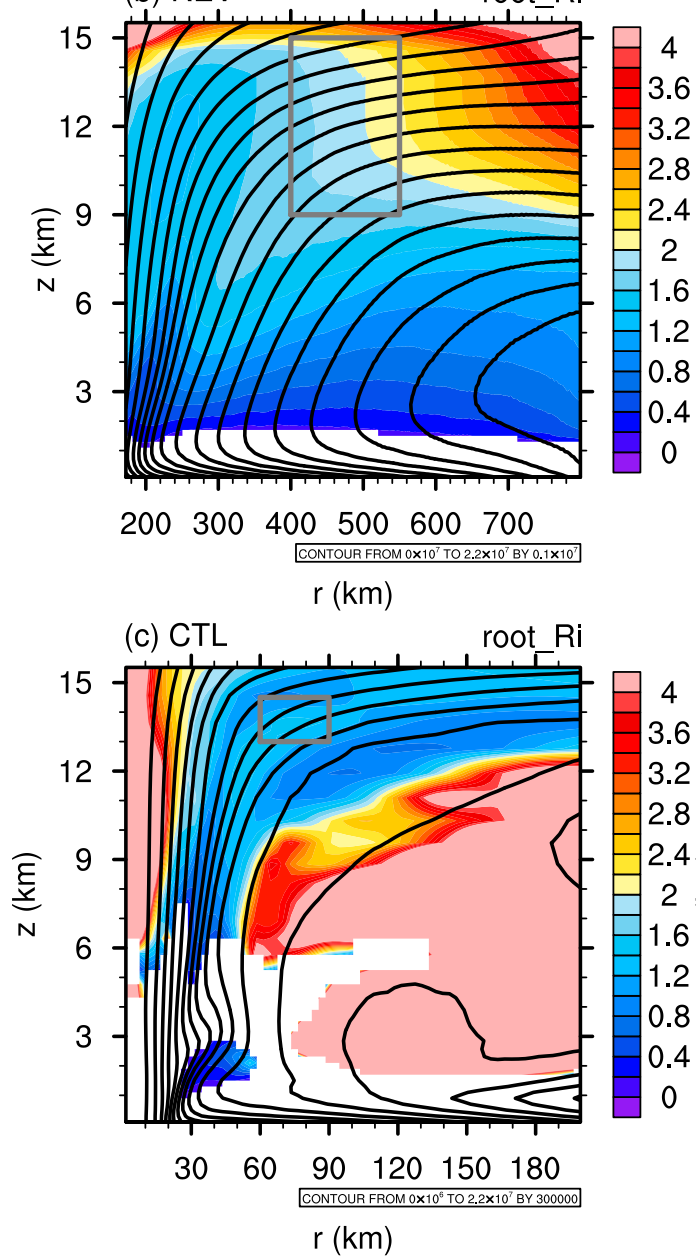

(d) DRY

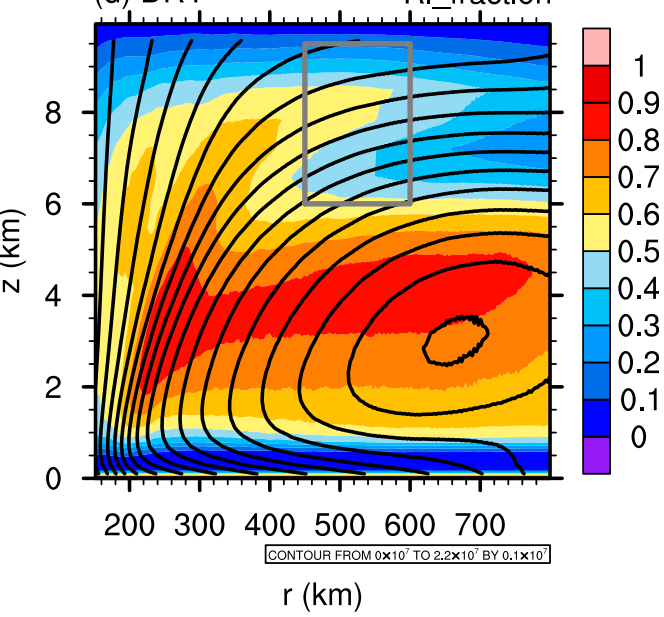

(e) REV

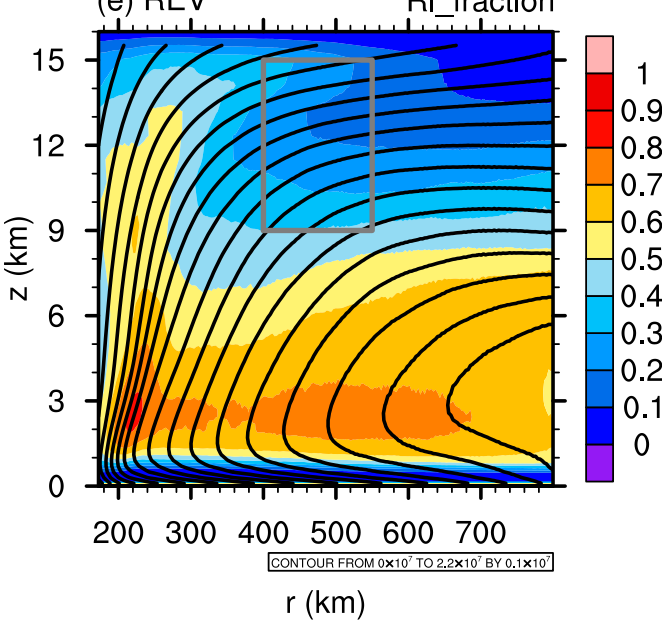

(f) CTL
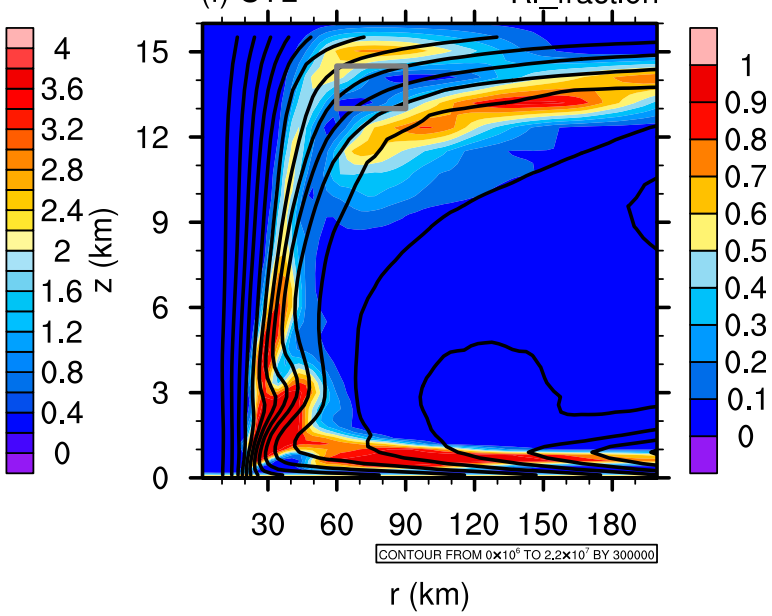

FIG. 6. (a)-(c) Square root of Ri (shaded) calculated from time- and azimuthal-mean fields during the steady state. Regions with negative values are masked in white. (d)-(f) Fraction (shaded) of grid points with $0<\mathrm{Ri}<1$ along an azimuth, where $\mathrm{Ri}$ is calculated by Eq. (9), during the steady state. Contour lines of $M$ (same interval as Figs. 4j-O) are overlaid. Gray boxes indicate analysis areas (see text). (top) DRY, (middle) REV, and (bottom) CTL. 
(a) DRY
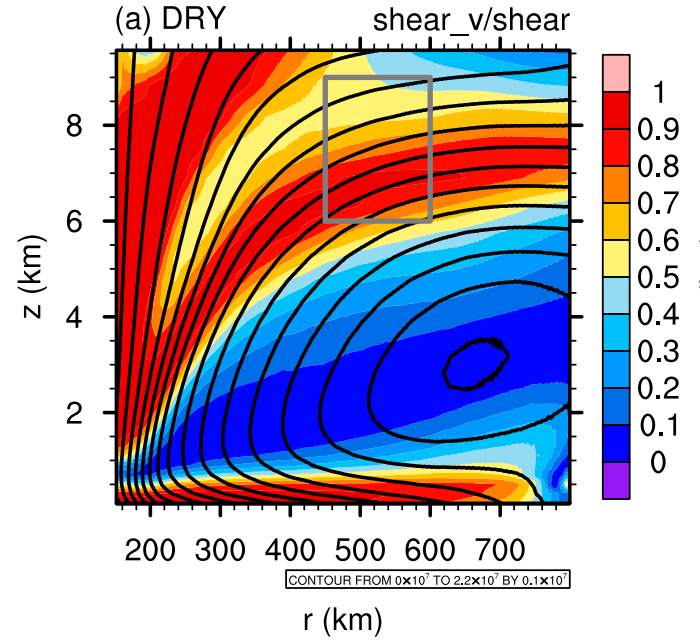

(b) REV

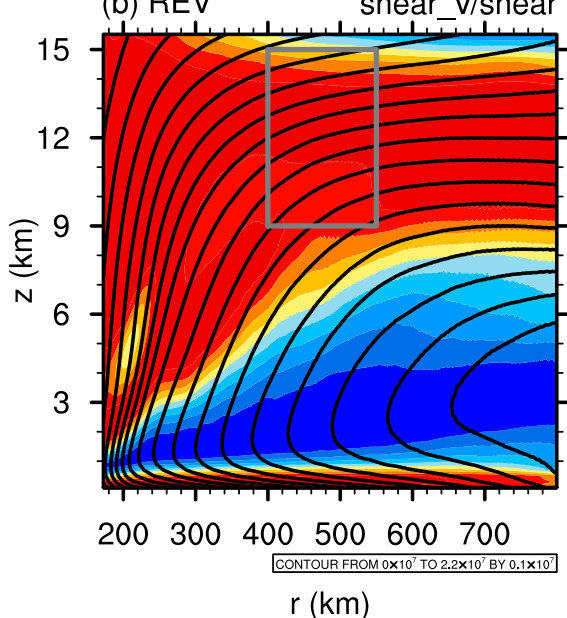

(c) CTL

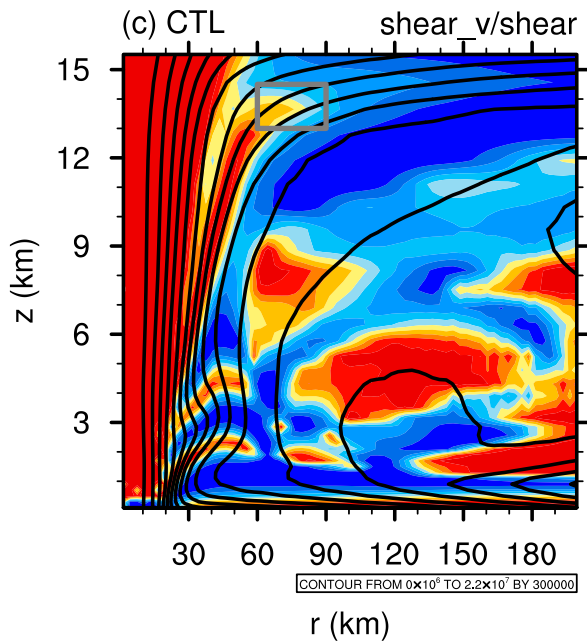

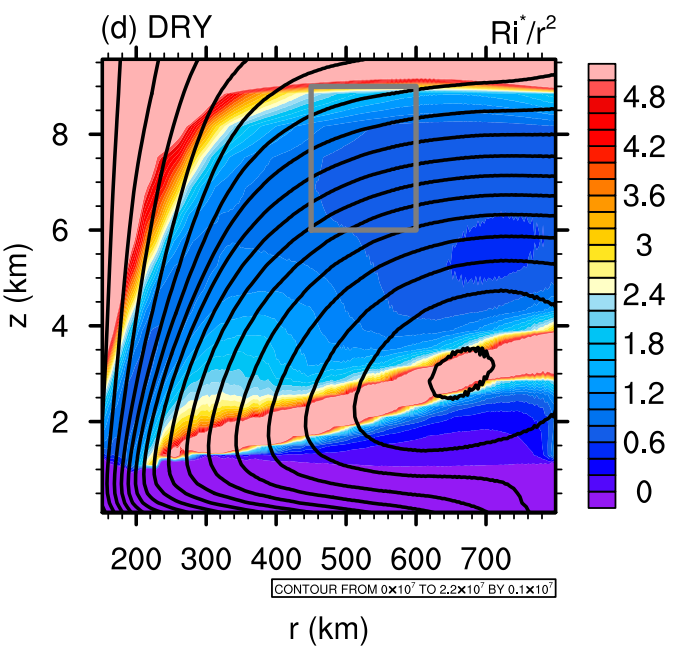
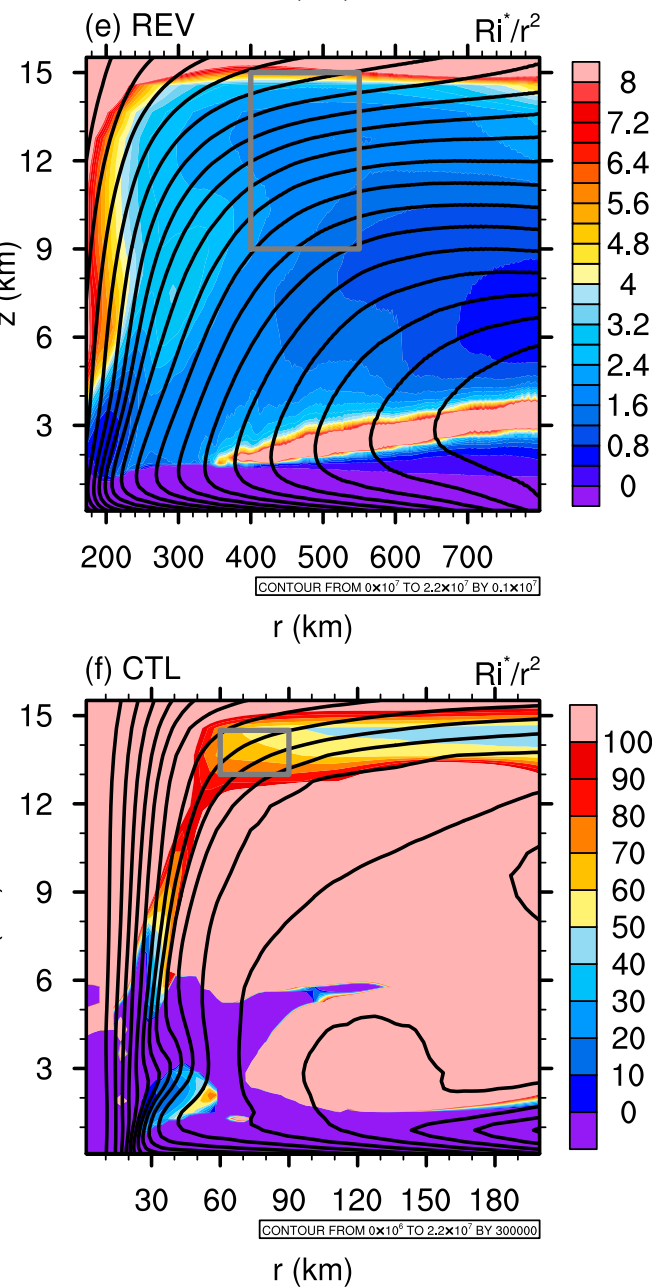

FIG. 7. (a)-(c) Ratio (shaded) of the time- and azimuthal-mean $(\partial v / \partial z)^{2}$ to $(\partial u / \partial z)^{2}+(\partial v / \partial z)^{2}$ during the steady state. (d)-(f) $\mathrm{Ri} * / r^{2}\left(10^{-11} \mathrm{~m}^{-2}\right.$; shaded) calculated from time- and azimuthal-mean fields during the steady state. Contour lines of $M$ (same interval as Figs. $4 \mathrm{j}-\mathrm{O}$ ) are overlaid. Gray boxes indicate analysis areas (see text). (top) DRY, (middle) REV, and (bottom) CTL. 
gray box, located at the inception of the outflow where slantwise neutrality is assumed to be most valid, and covering the depth of the middle and upper parts of the outflow. As the derivation of Eq. (5) neglects the vertical shear of radial wind $u$, it is necessary to first examine the ratio of $(\partial v / \partial z)^{2}$ to $(\partial u / \partial z)^{2}+(\partial v / \partial z)^{2}$, with $v$ the tangential wind (Figs. $7 \mathrm{a}-\mathrm{c}$ ). It is evident that this ratio is typically large only in the middle of the outflow, and becomes small in upper and lower edges of the outflow, due to a large vertical shear of radial wind, especially in DRY. An outward decrease of the ratio in CTL is also noted. For a more accurate evaluation of Eq. (5), $\mathrm{Ri}^{*} / r^{2}$, with $\mathrm{Ri}^{*}=N_{m}^{2} /(\partial v / \partial z)^{2}$ is calculated from the time and azimuthal mean fields (Figs. 7d-f). It is noted that $\mathrm{Ri}^{*} / \mathrm{r}^{2}$ does not vary much in a fairly large area of the outflow layer. We use the average of $\mathrm{Ri}^{*} / r^{2}$ in the gray box (Figs. $7 \mathrm{~d}-\mathrm{f}$ ) to approximate $\mathrm{Ri} / r_{t}^{2}$ in Eq. (5) to calculate the theoretical wind profile.

The theoretical tangential wind profile from ER11 was evaluated in Fig. 8. This analysis is designed to test the role of varying $T_{o}$ [Eq. (5)] in modulating tangential wind at the top of the boundary layer. As the ascent region becomes hundreds of kilometers wide, a numerical solution of ER11 can be calculated (see appendix A for details) rather than the analytical one, which neglects the effect of Coriolis parameter. The theoretical wind profile applies generally well in REV and CTL, but to a lesser extent in DRY probably related to an effect of the three-dimensional geometry, different from the axisymmetric assumption of ER11. Note that ER11 neglected the effect of $q_{t}$, which was incorporated (see appendix B) to calculate a modified tangential wind profile for REV (Fig. 8b). Wind speed was reduced by $\sim 5 \mathrm{~m} \mathrm{~s}^{-1}$ at $r_{m}$ and in better agreement with simulated wind profiles, especially the gradient wind profile, in REV with the effect of $q_{t}$ included (Fig. 8b).

The above analysis indicates that ER11 theory applies well in dry-type TCs in terms of both basic structures and boundary layer closure. Note that the intensity has reached its upper bound at about $130 \mathrm{~h}$ in DRY, but the size keeps increasing (Fig. 2). This not only suggests that steady-state intensity and size are independent, as predicted by the analytical solution of ER11 (see below), but also provides an opportunity to explore some implicit properties of the ER11 model.

Substituting Eqs. (2)-(4) into Eq. (1) and including viscous dissipation in Eq. (3) results in (Bister and Emanuel 1998)

$$
v_{b}^{2} \cong \frac{c_{k}}{c_{D}} \frac{T_{b}-T_{o}}{T_{o}}\left(k_{0}^{*}-k\right) .
$$
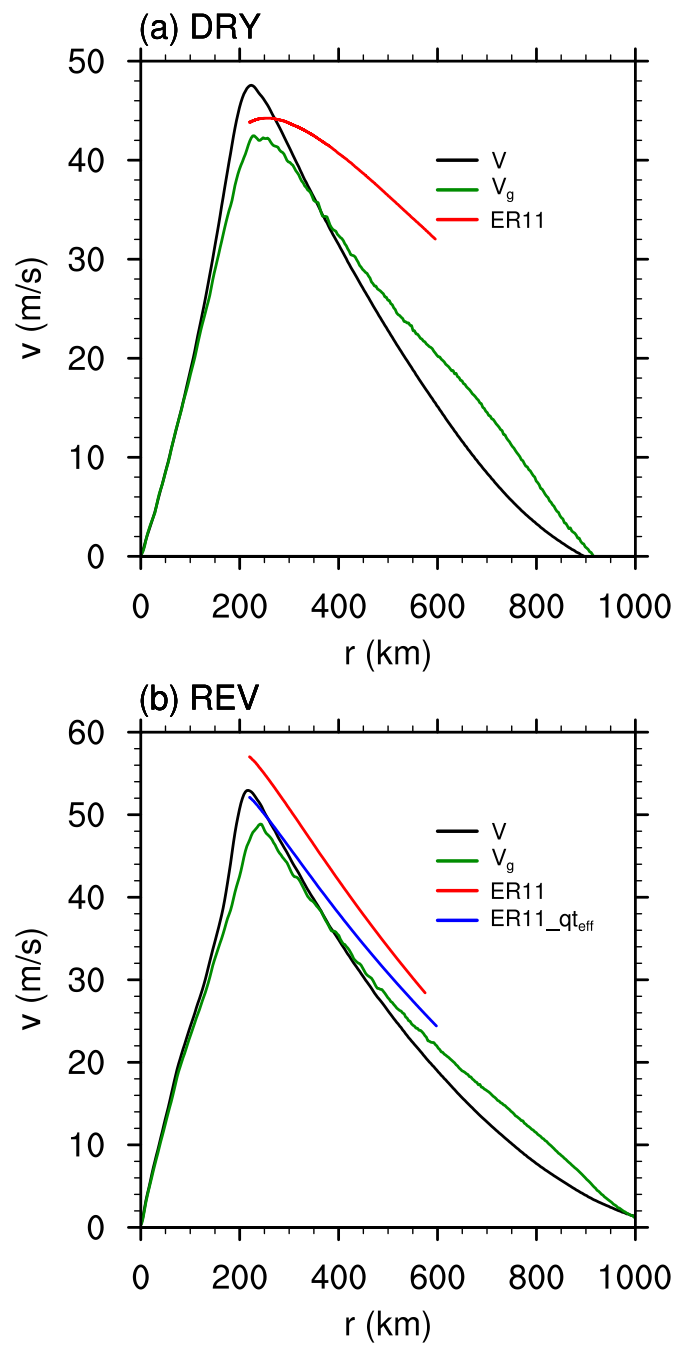

(c) CTL

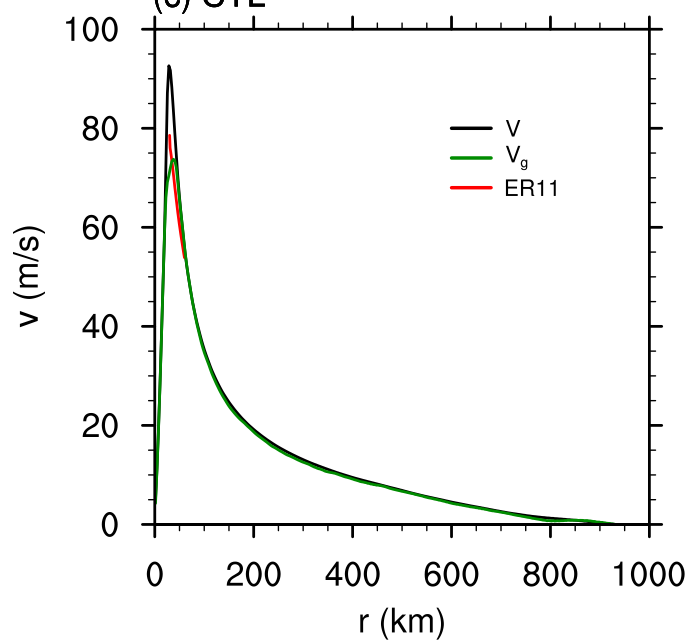

FIG. 8. Comparison of theoretical tangential wind profile $\left(\mathrm{m} \mathrm{s}^{-1}\right.$; red) at the top of the boundary layer from ER11 with actual and gradient tangential wind profiles at $1 \mathrm{~km}$ of altitude $\left(\mathrm{m} \mathrm{s}^{-1}\right.$; black and green, respectively) in (a) DRY, (b) REV, and (c) CTL during the steady state. The theoretical wind profile $\left(\mathrm{m} \mathrm{s}^{-1}\right)$ including the dependence of total water mixing ratio for REV is shown in blue. 
Following ER11, under the eyewall ascent area, the airsea enthalpy difference $k_{0}^{*}-k$ decreases inward from an ambient value. This is evident in Figs. 9a and $9 \mathrm{~b}$ for drytype TCs, but not for CTL and may contribute to a more intense TC by a larger $k_{0}^{*}-k$ at $r_{m}$. ER11 predicted that the maximum wind is a function of environmental parameters alone [their Eq. (41)]. From Eq. (12), this requires $\left(k_{0}^{*}-k\right)$ to be a constant with varying $r_{m}$ as $\left(T_{b}-\right.$ $\left.T_{o}\right) / T_{o}$ is also approximately constant at $r_{m}$. This point is evident in DRY (Fig. 9a) as the contours of $\left(k_{0}^{*}-k\right)$ and surface tangential wind are approximately parallel. The same is also true in REV when it becomes quasi steady after $350 \mathrm{~h}$.

As a part of the analytical solution, ER11 derived in their Eq. (42) that $r_{m}$ is approximately proportional to $r_{0}^{2}$, where $r_{0}$ is the outer radius with vanished gradient wind:

$$
r_{m} \simeq\left(\frac{1}{2}\right)^{3 / 2} \frac{f r_{0}^{2}}{\sqrt{\left(T_{b}-T_{t}\right)\left(s_{0}^{*}-s_{e}^{*}\right)}}
$$

where $T_{t}$ may be approximated by the tropopause temperature (here taken as the lowest value in the initial sounding of each experiment), $s_{0}^{*}$ the saturation entropy at SST, and $s_{e}^{*}$ the saturation entropy of environmental near surface air; $T_{b}$ is taken as the temperature at $r_{m}$ at $1 \mathrm{~km}$ of altitude. Using the approximation $s_{0}^{*}-s_{e}^{*} \simeq\left(k_{0}^{*}-k_{e}^{*}\right) / \mathrm{SST}$, with $k_{0}^{*}-k_{e}^{*}=13 \mathrm{~kJ} \mathrm{~kg}^{-1} \mathrm{ac}-$ cording to Fig. $9 \mathrm{a}$ and $T_{b}-T_{t}=89 \mathrm{~K}$, Eq. (13) is supported in DRY after $130 \mathrm{~h}$ when the maximum intensity is reached (Fig. 10), though here the outer radius $r_{0}$ is measured by $r_{4}$ for a better fit. In REV, size has become quasi steady after reaching maximum intensity. Using $r_{4}$ as the outer radius and taking $k_{0}^{*}-k_{e}^{*}=18 \mathrm{~kJ} \mathrm{~kg}^{-1}$ from Fig. 9b, Eq. (13) gives a $r_{m}$ of $\sim 187 \mathrm{~km}$, comparable to the actual $r_{m}(\sim 220 \mathrm{~km})$ in REV. However, in CTL, the environment is not saturated and the wide subsidence region is clearly not slantwise neutral, so the relation of $r_{m}$ and $r_{0}$ in ER11 cannot be realized. As expected, taking $k_{0}^{*}-k_{e}^{*}=20 \mathrm{~kJ} \mathrm{~kg}^{-1}$ gives a poor prediction of $r_{m}$ of $\sim 86 \mathrm{~km}$, about 3 times of the actual value in CTL. Qualitatively similar findings are also seen in Chavas et al. (2015), Chavas and Lin (2016) and CC19.

In this section, a systematic evaluation of ER11 is performed. Not only the basic assumptions and relations, but also the wind profile, deductions and analytical relation between $r_{m}$ and $r_{0}$ are shown applicable to DRY-type TCs. Overall, the ER11 model captures the important physics of dry-type TCs. The evaluation also indicates an equivalent nature of DRY and REV in the ascent region. Overall, it can be argued that dry-type TCs behave like the prototypes of the ER11 model with typical moist TCs acting as extreme cases.
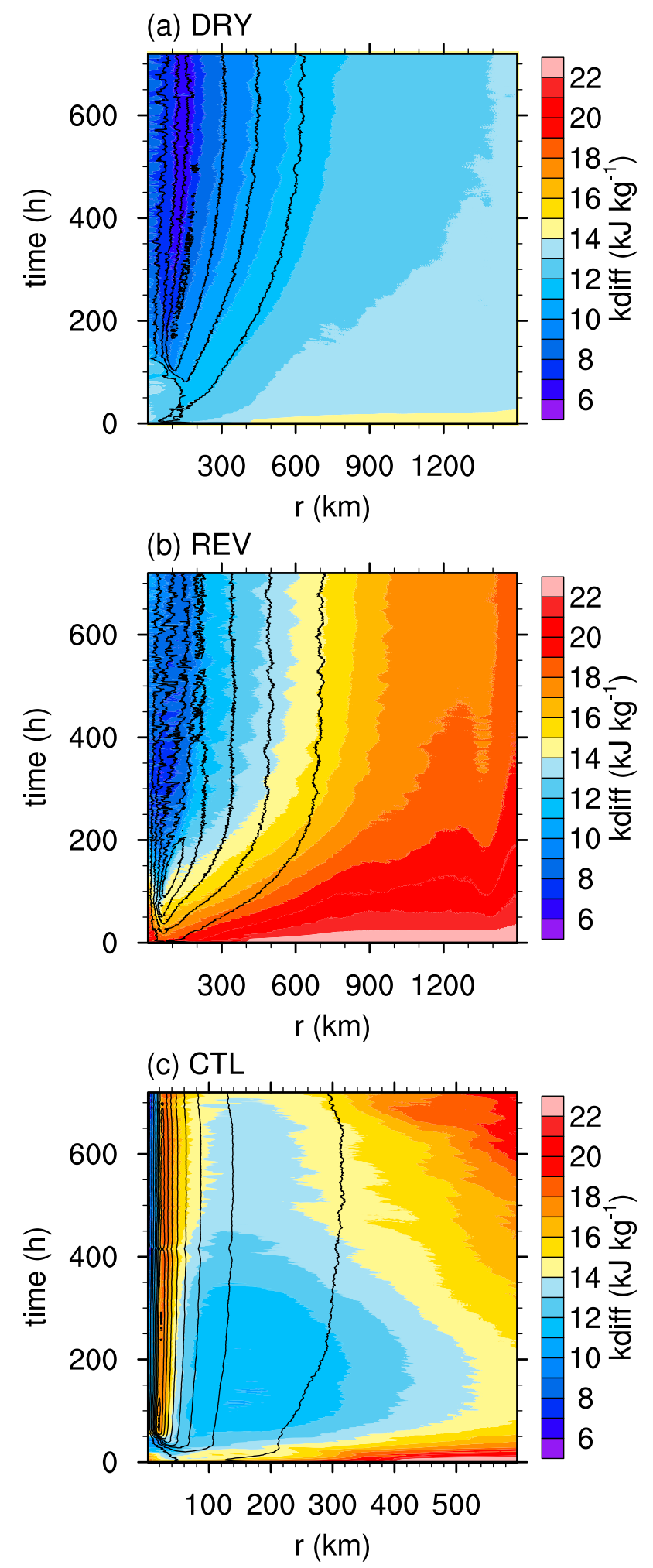

FIG. 9. Radius-time sections of air-sea enthalpy difference ( $\mathrm{kJ} \mathrm{kg}^{-1}$; shaded) and surface tangential wind ( $\mathrm{m} \mathrm{s}^{-1}$; contours, with an interval of $10 \mathrm{~m} \mathrm{~s}^{-1}$ starting from $10 \mathrm{~m} \mathrm{~s}^{-1}$ ) in (a) DRY, (b) REV, and (c) CTL. The air-sea enthalpy difference is calculated from azimuthal-mean variables. 


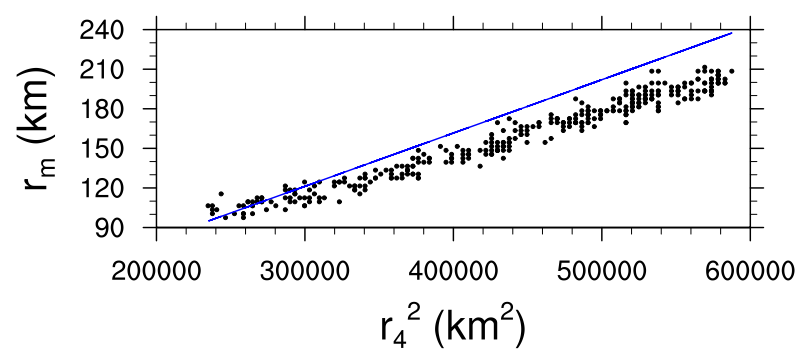

FIG. 10. Scatterplot of $r_{m}(\mathrm{~km})$ and $r_{4}^{2}\left(\mathrm{~km}^{2}\right)$ in DRY after $130 \mathrm{~h}$. The blue line is the prediction of $r_{m}$ by Eq. (13) using $r_{4}$ as the outer radius. Scatter points are obtained from hourly model output.

\section{b. Different widths of the ascent and $r_{m}$}

Though ER11 catches the physics of the TC structure in the eyewall region, it does not constrain the width of the eyewall. As evident in Fig. 3 and Table 2, the ascent region in CTL is much narrower than REV, though they are initiated from very similar initial conditions. When eyewall ascent is established, compensating subsidence is induced just outside of the eyewall. As a result, the environment would become drier as the upper-level air, whose water has been mostly precipitated, descends. At the early stage $(10 \mathrm{~h})$, the whole troposphere is nearly saturated (Fig. 11a) with deep convections initiated and a nascent secondary circulation formed. At $20 \mathrm{~h}$, the secondary circulation strengthens and the radial width of the ascending region reaches $150 \mathrm{~km}$, with significant subsidence of dry air in the upper troposphere just outside the ascending region. At $30 \mathrm{~h}$, an upper-level inflow layer has been established, which brings upper-level subsaturated air inward to the convective region and decreases $\mathscr{H}$ in the midtroposphere. Correspondingly, the width of the ascending region shrinks significantly to $\sim 50 \mathrm{~km}$ in the lower troposphere. At 40-160 h (Figs. 11d-f), dry air becomes more dominant outside the eyewall and $\mathscr{H}$ becomes $<50 \%$ immediately above $1 \mathrm{~km}$ of altitude from 100 to $200 \mathrm{~km}$ of radius at $160 \mathrm{~h}$. The width of ascent remains small with decreased vertical mass flux indicated by a decreased maximum value of streamfunction compared to $20 \mathrm{~h}$.

The above process may be explained by the role of midlevel dry air to weaken the strength of deep convection by reduced buoyancy through entrainment (James and Markowski 2010; Smith and Montgomery 2012; Kilroy and Smith 2012; Freismuth et al. 2016). This mechanism does not work in the outer region in dry-type TCs due to the approximate conservation of entropy and thus a wide ascending region can be established.

The reason for the difference of $r_{m}$ between dry-type and CTL TCs is discussed in the following. For what controls $r_{m}$ in dry-type TCs, we refer to Emanuel's theory because of their wide ascent regions. From Eq. (12), with $\left(k_{0}^{*}-k\right)$ decreasing inward and $\left(T_{b}-T_{o}\right) / T_{o}$ increasing inward, there would be a certain radius where gradient wind is maximum. Analytically, $r_{m}$ is determined by the outer radius as shown in the last section. Due to the existence of a wide subsidence region and a narrow ascent region, ER11 does not link $r_{m}$ and the outer radius well in CTL. Nonetheless, a complete model of radial wind profile, which merged an outer region model (Emanuel 2004) with ER11 model, did successfully predict $r_{m}$ to be quite small (tens of kilometers) from a given reasonable outer radius $(\sim 900 \mathrm{~km}$; Chavas et al. 2015; Chavas and Lin 2016). However, currently, what determines the outer radius is not well known, though a dominant length scale of the outer radius is $V_{p} / f$ on an $f$ plane (Chavas and Emanuel 2014), where $V_{p}$ is the potential intensity, whereas in the existence of $\beta(=d f / d y)$, the outer radius is proposed to follow the smaller one of $V_{p} / f$ and the Rhines scale (Rhines 1975; Chavas and Reed 2019).

\section{c. Relative width of subsidence region and subsidence velocity}

The distinctive relative widths of the ascending and descending region in dry-type and typical TCs (Fig. 3) may be related to the vertical gradient of entropy in the descending region. First, we discuss two parameters controlling the relative widths. By integrating Eq. (10) from $r_{\psi_{0}}$ to $r_{\psi_{\max }}$, a relation between $r_{\psi_{0}}$ and $r_{\psi_{\max }}$ (Table 2) can be derived:

$$
\left(\frac{r_{\psi_{0}}}{r_{\psi_{\max }}}\right)^{2}=\frac{-2 \psi_{\max } / r_{\psi_{\max }}^{2}}{\rho_{0} w_{\mathrm{rad}}}+1,
$$

where $\rho_{0}$ and $w_{\text {rad }}$ are air density and subsidence velocity, both assumed to be constant at a certain altitude in Eq. (14). Note that $r_{\psi_{0}}-r_{\psi_{\max }}$ measures the radial width of the descent at a certain altitude. From Eq. (14), we see that $\psi_{\max } /\left(\rho_{0} r_{\psi_{\max }}^{2}\right)$ and $w_{\text {rad }}$ are two important parameters controlling the ratio of $r_{\psi_{0}}$ to $r_{\psi_{\max }}$, with $2 \psi_{\max } /\left(\rho_{0} r_{\psi_{\max }}^{2}\right)=w_{u}$, where $w_{u}$ is the mean vertical velocity in the eye and eyewall. With a small $\psi_{\max } /\left(\rho_{0} r_{\psi_{\max }}^{2}\right)$ or large $w_{\text {rad }}, r_{\psi_{0}}$ would be close to $r_{\psi_{\max }}$, that is, the relative width of the descending region becomes smaller. A crude approximation of Eq. (14) gives that $w_{\text {rad }}$ in dry-type TCs should be $\sim 6$ times of that in CTL, similar to that in Fig. 13b. The quantity $\psi_{\max } /\left(\rho_{0} r_{\psi_{\max }}^{2}\right)$ is related to the radially varying ascending velocity and is difficult to determine, though it may be conceptually diagnosed from a twodimensional pressure field from ER11 in the ascending region. The controlling mechanism of $w_{\text {rad }}$ may be more conveniently illustrated by an entropy budget in the following. 
(a) $10 \mathrm{~h}$

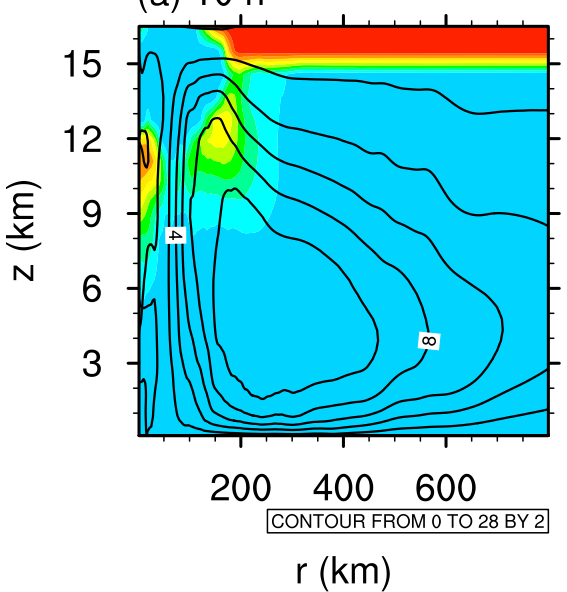

(c) $30 \mathrm{~h}$

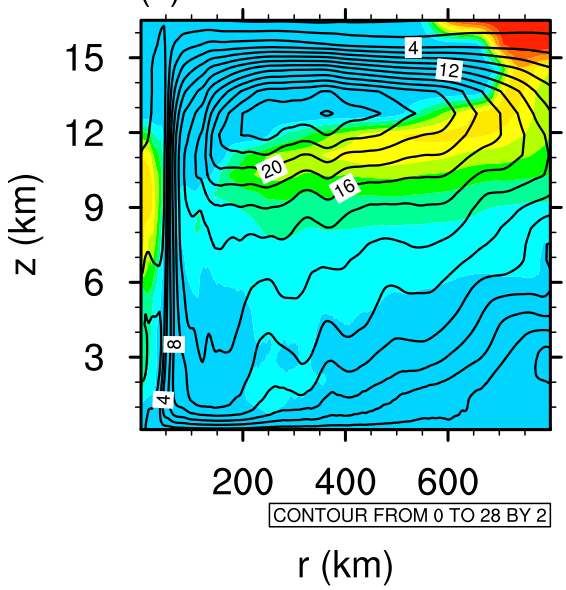

(e) $80 \mathrm{~h}$

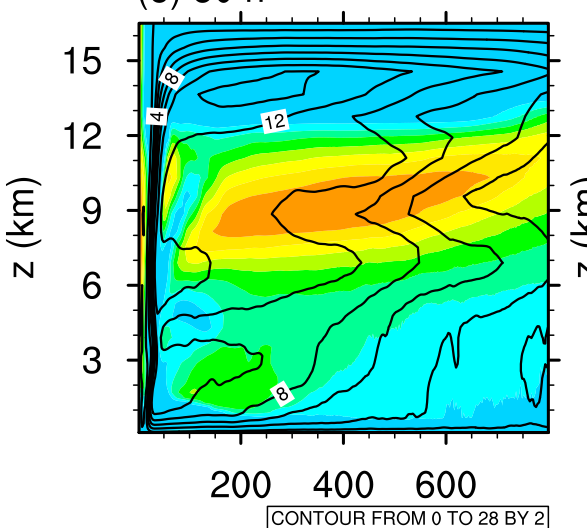

$\mathrm{r}(\mathrm{km})$ (b) $20 \mathrm{~h}$

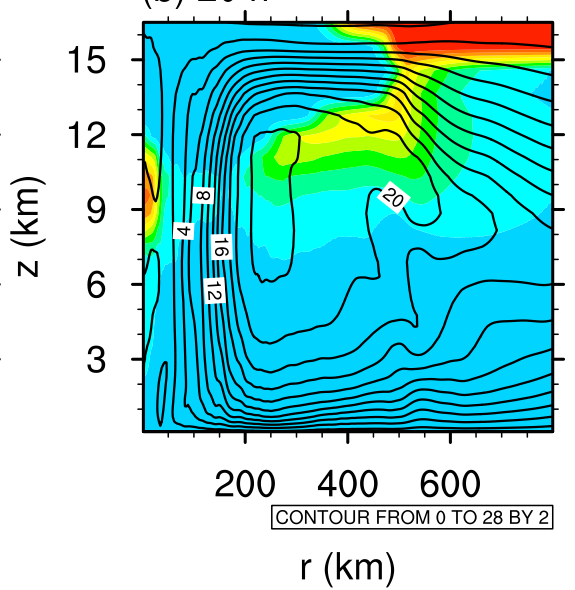

(d) $40 \mathrm{~h}$

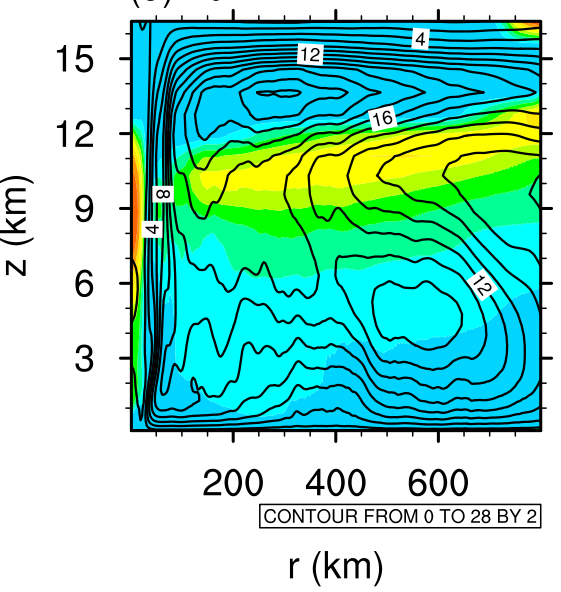

(f) $160 \mathrm{~h}$

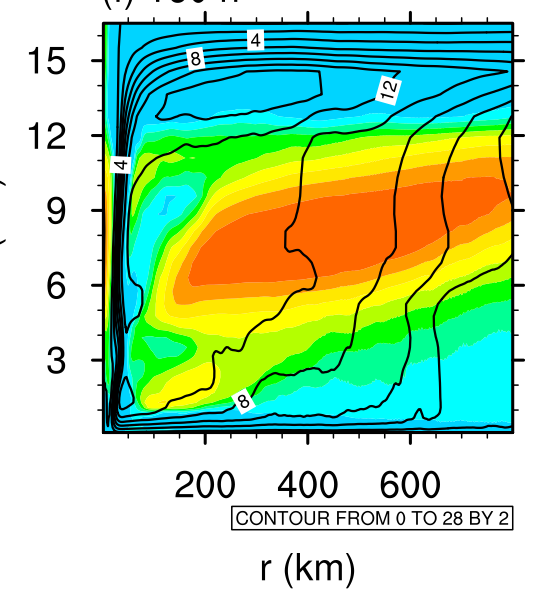

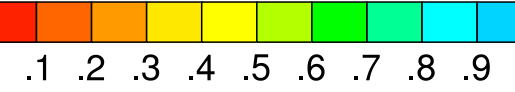

$\mathrm{RH}$

FIG. 11. Height-radius sections of relative humidity (calculated from azimuthal-mean fields; shaded) and mass streamfunction (contours, with an interval of $2 \times 10^{8} \mathrm{~kg} \mathrm{~s}^{-1}$ ) of CTL at (a) 10, (b) 20, (c) 30, (d) 40, (e) 80, and (f) $160 \mathrm{~h}$. 
In the subsidence region, presumably the entropy budget [Eq. (7)] is dominated by the vertical advection and radiative cooling. Thus, subsidence velocity may be diagnosed by balancing the two terms:

$$
\overline{w_{\text {diag }}}=\overline{\delta S_{\text {rad }}} / \frac{\partial \bar{s}}{\partial z}
$$

where $w_{\text {diag }}$ is a diagnosed subsidence velocity corresponding to $w_{\mathrm{rad}}$ and $\partial s / \partial z$ the vertical gradient of entropy. Note that the vertical mean advection $(-\bar{w} \partial \bar{s} / \partial z)$ is used to approximate total vertical advection in Eq. (15).

Vertical distribution of azimuthally averaged entropy budget averaged in the subsidence region $(400-800 \mathrm{~km})$ during the steady state for CTL is shown in Fig. 12a. It is evident that in the subsidence region, the dominant terms are vertical advection and radiative cooling, especially above $5 \mathrm{~km}$ of altitude. The diagnosed subsidence velocity (Fig. 12d) compares well with the actual subsidence velocity above $5 \mathrm{~km}$ of altitude, giving a good support of Eq. (15) and demonstrating the balance between vertical mean entropy advection and entropy sink from radiative cooling. Slight underestimates of subsidence velocity above $12 \mathrm{~km}$ is associated with the nonnegligible horizontal advection there. The subsidence velocity is $\sim 0.5-1.3 \mathrm{~cm} \mathrm{~s}^{-1}$ in CTL in the midto upper troposphere. Below $5 \mathrm{~km}$, vertical advection begins to change sign downward with significantly increased horizontal advection, consistent with the lowertropospheric entropy structure (Fig. 3c). These features deviate from the assumed balance in Eq. (15), and thus $\overline{w_{\text {diag }}}$ does not work well in the lower troposphere.

The entropy budget in the subsidence region in drytype TCs (Figs. 12b,c) indicates that horizontal and vertical advection and radiative cooling dominate, with the relative importance of each term varies with height. Note that subsidence region for analysis is $800-1100 \mathrm{~km}$ of radius for both DRY and REV. Nonetheless, the diagnosed subsidence velocity still compares fairly well with the actual subsidence velocity in the upper and midtroposphere (Figs. 12e,f). Some overestimates are associated with a relatively smaller contribution of vertical mean advection to the total vertical advection, and a relatively larger contribution of vertical eddy advection $\left(-\overline{w^{\prime} \partial s^{\prime} / \partial z}=\overline{\mathrm{ADV}_{v}}+\bar{w} \overline{\partial s / \partial z}\right)$. In the lower troposphere, the diagnosed subsidence velocity is substantially larger than the actual subsidence velocity as the vertical eddy advection becomes significant (not shown), due to active convective cells.

Above $6 \mathrm{~km}$ of altitude where Eq. (15) is crudely valid, the subsidence velocity in dry-type TCs can be $\sim 11$ times larger than the typical TC with the corresponding azimuthally averaged vertical entropy gradient $\sim 11$ times smaller than the typical TC (Fig. 13), demonstrating the important role of vertical entropy gradient. The entropy sink from radiative cooling does not differ much (except between 10 and $12 \mathrm{~km}$ of altitude in CTL) because it is capped at $2 \mathrm{~K} \mathrm{day}^{-1}$ in all experiments.

Different vertical gradients of entropy between REV and CTL is explained as follows. The vertical gradient of entropy increases significantly reaching the tropopause due to a self-stratification in the outflow (Fig. 13a). From 6 to $12 \mathrm{~km}$ of altitude, the lapse rate has become close to a moist adiabatic lapse rate in REV and CTL, and the difference of vertical entropy gradient mainly comes from a lack of water in CTL, where the vertical velocity difference also becomes most significant. The vertical gradient of entropy may be obtained following Eq. (6) (Emanuel 1994). A major difference between REV and CTL is that water vapor mixing ratio increases downward due to evaporation of liquid water in REV, while it remains nearly constant in CTL because there is no liquid water to evaporate. This difference is a consequence of whether or not allowing hydrometeors to fall in the atmosphere.

Alternatively, as the moist processes are suppressed in the subsidence region in CTL, we may substitute $\overline{\partial s / \partial z}$ by $\overline{\partial s_{d} / \partial z}$ in Eq. (15), where $s_{d}$ is the dry entropy, as suggested in Emanuel (2004), so that

$$
\overline{w_{\text {diag } *}}=\overline{\delta S_{\mathrm{rad}}} / \frac{\partial \overline{s_{d}}}{\partial z},
$$

in which $\partial \overline{s_{d}} / \partial z \approx c_{p} \partial \ln \bar{\theta} / \partial z$. The vertical profiles of $\overline{w_{\text {diag }} *}$ from Eq. (16) compared well to $\bar{w}$ from 2 to $12 \mathrm{~km}$ (Fig. 14). Compared to $\overline{w_{\text {diag }}}$ from Eq. (15), $\overline{w_{\text {diag* }}}$ also predicts the subsidence in lower troposphere well. This is because while $\partial \bar{S} / \partial z$ becomes negative in the lower troposphere (Fig. 3c), $\partial \overline{s_{d}} / \partial z$ still remains positive associated with the balance indicated by Eq. (16).

In a broader sense, $\partial \overline{s_{d}} / \partial z$ corresponds to the stability of subsaturated air (subsidence in CTL and DRY) and $\overline{\partial s^{*} / \partial z}$ corresponds to the stability of saturated air (subsidence in REV) (Emanuel 1994). Thus, the atmosphere is much more stable to adiabatic vertical motions in CTL than in dry-type TCs, consistent with the argument in CC19. In this manner, given a small radiative cooling rate, the descent is very slow in CTL because the descent is strongly diabatically forced. This also creates a fundamental asymmetry of fast ascent and slow descent in CTL, manifested in a convective moist atmosphere in general (Bjerknes 1938). For mass continuity, the subsidence must occupy a much larger area than the ascent so that the net vertical mass flux would be zero for the whole TC in CTL (Fig. 3). 

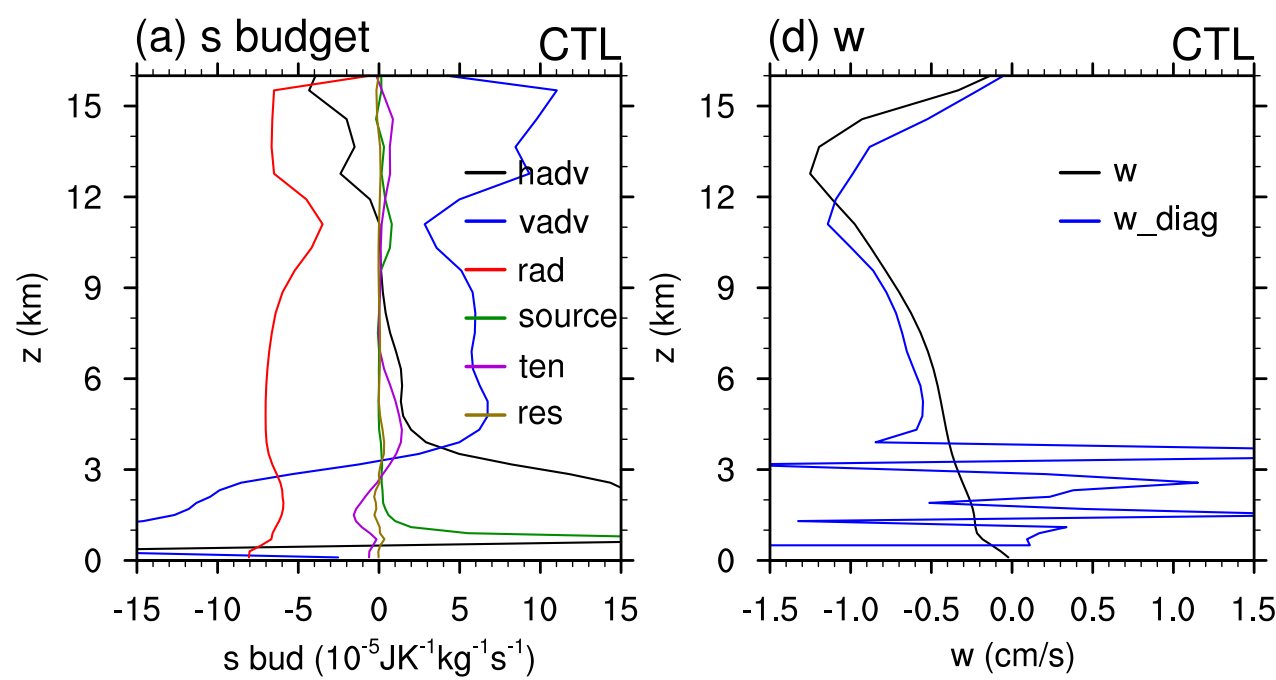

(b) s budget
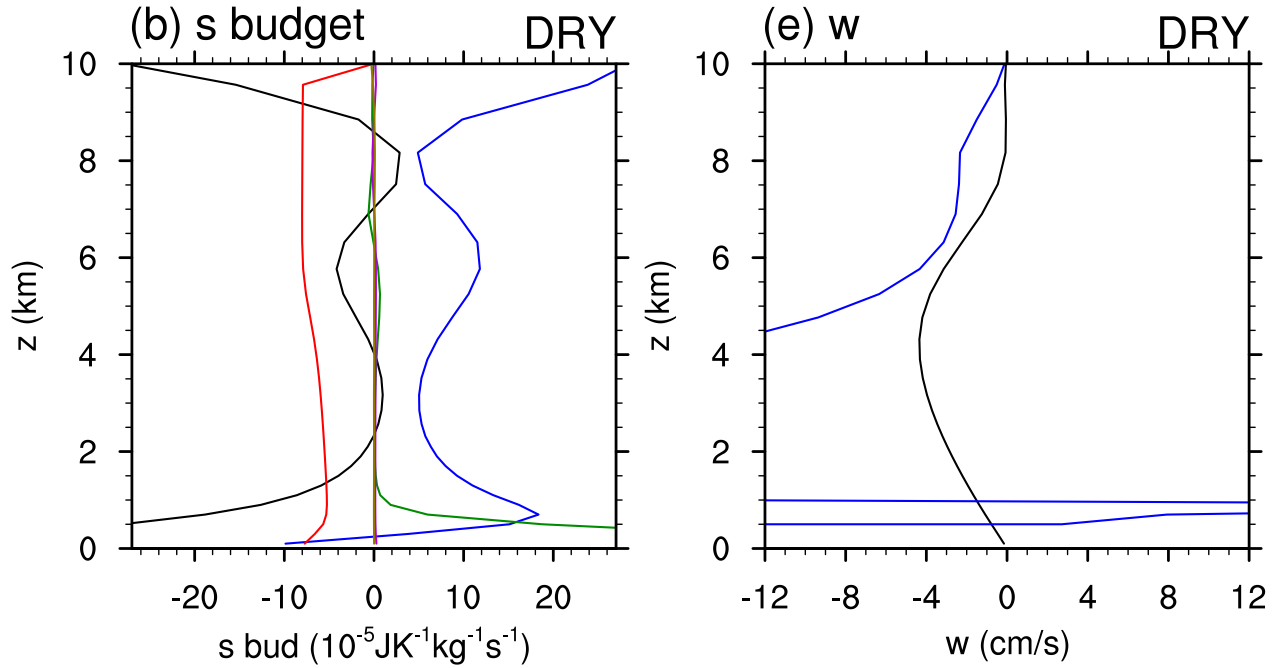

(c) s budget
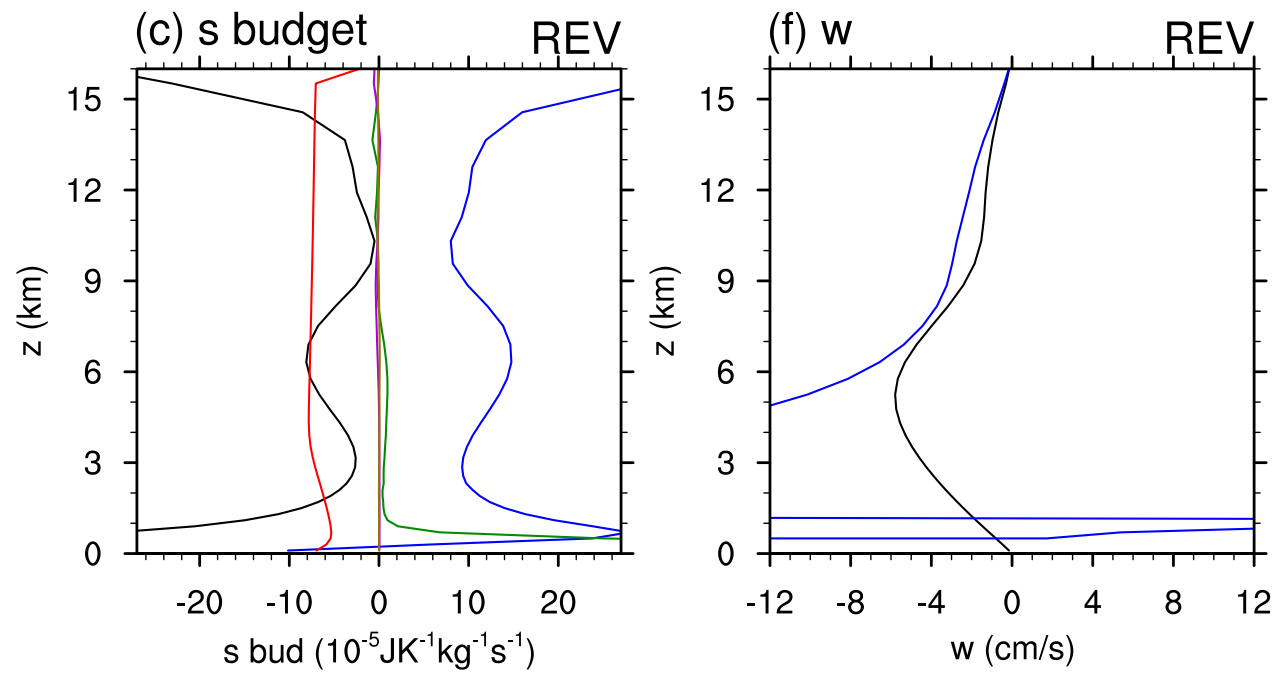

FIG. 12. (a)-(c) Vertical distribution of azimuthally averaged entropy budgets [Eq. (7); $10^{-5} \mathrm{~J} \mathrm{~K}^{-1} \mathrm{~kg}^{-1} \mathrm{~s}^{-1}$ ] and (d)-(f) comparison between diagnosed subsidence velocity [Eq. (15); $\mathrm{cm} \mathrm{s}^{-1}$, blue] and actual vertical velocity ( $\mathrm{cm} \mathrm{s}^{-1}$; black) in (top) CTL, (middle) DRY, and (bottom) REV during the steady state. Values are radially averaged from 400 to $800 \mathrm{~km}$ in CTL and from 800 to $1100 \mathrm{~km}$ in DRY and REV. Terms in (a)-(c) are $\mathrm{ADV}_{h}$ (black), $\mathrm{ADV}_{v}$ (blue), $\delta S_{\text {rad }}$ (red), sum of other source/sink terms $\delta S_{\text {sflx }}+\delta S_{\text {wflx }}+\delta S_{\text {irr }}$ (green), local entropy tendency (violet), and the residual between the local tendency and the sum of all terms (gold). 
(a) $d s / d z$

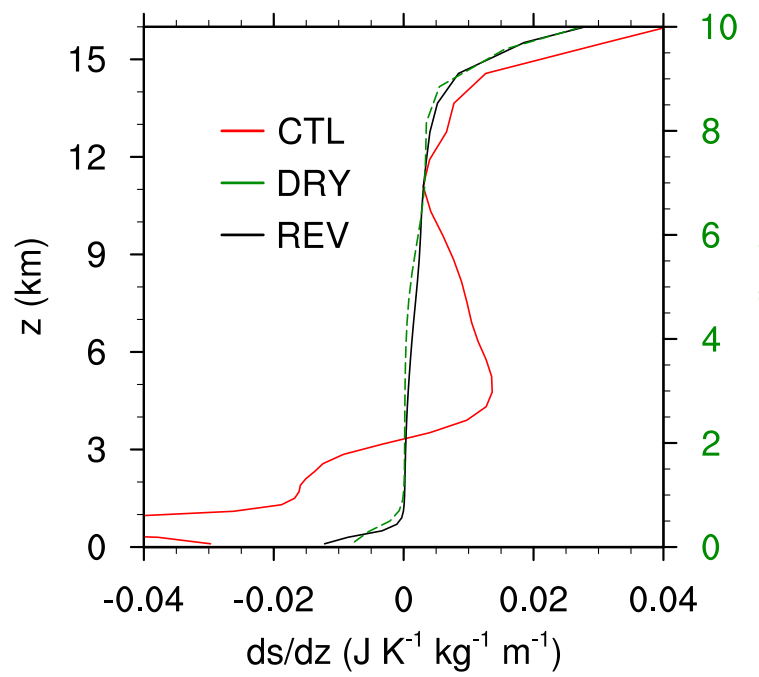

(b) w

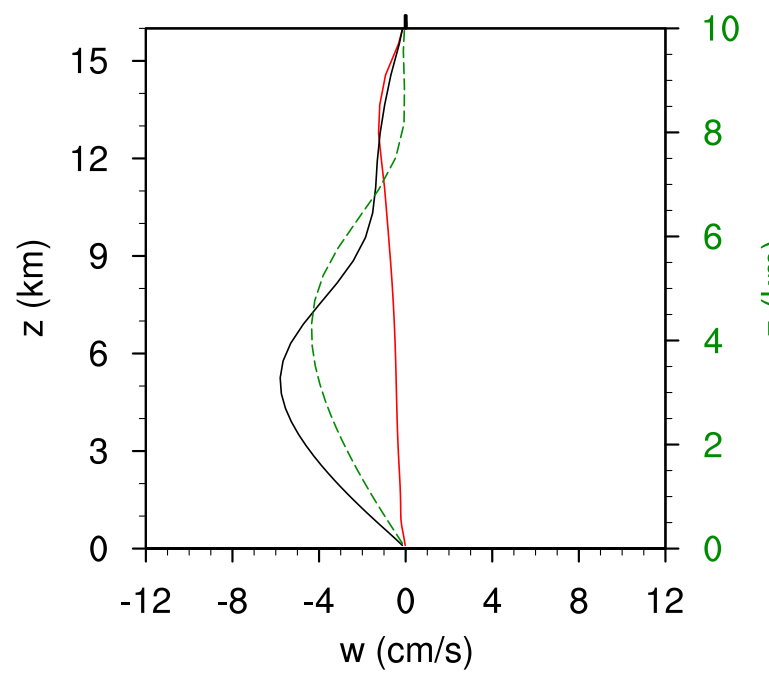

FIG. 13. Vertical profiles of azimuthal-mean (a) vertical gradient of entropy $\left(\mathrm{J} \mathrm{K}^{-1} \mathrm{~kg}^{-1} \mathrm{~m}^{-1}\right)$ and (b) vertical velocity $\left(\mathrm{cm} \mathrm{s}^{-1}\right)$ in CTL (red), DRY (green), and REV (black) during the steady state. Values are radially averaged from 400 to $800 \mathrm{~km}$ for CTL and from 800 to $1100 \mathrm{~km}$ for DRY and REV. The height coordinate for DRY is to the right axis.

In contrast, the descent in dry-type TCs can be fast with a smaller stability. The entropy of the descent is also nearly conserved, equivalent to the ascent. This helps explain nearly mirror image of the ascent and descent in dry-type TCs (Fig. 3). The mirror structure is also seen in the vertical: in the ascent region, buoyancy is produced via input of entropy by the inflow layer near the surface, whereas in the descent region buoyancy is produced in the outflow layer in the upper troposphere

\section{(a) w}

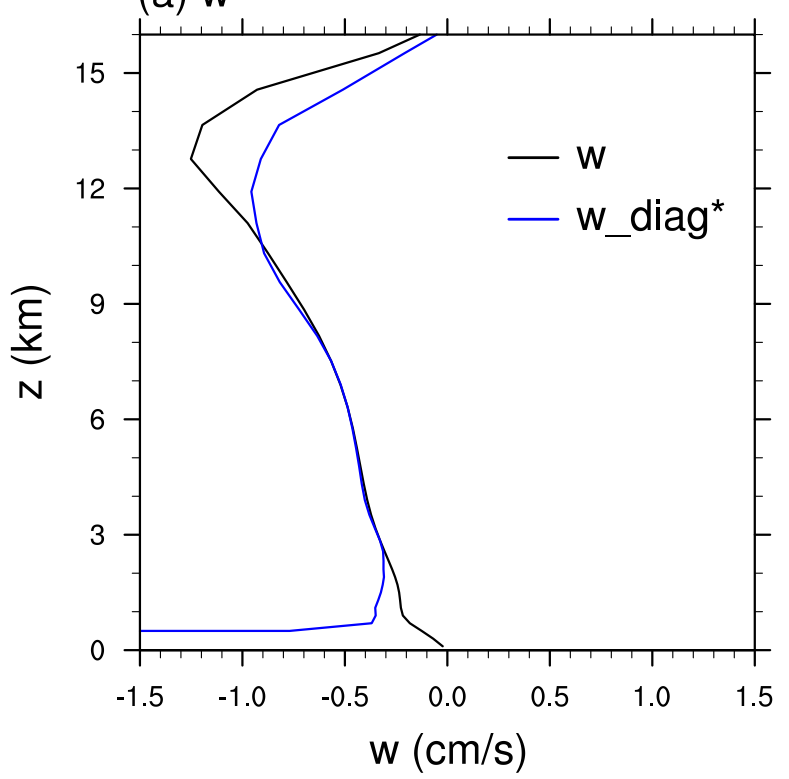

(b) $d s_{d} / d z$

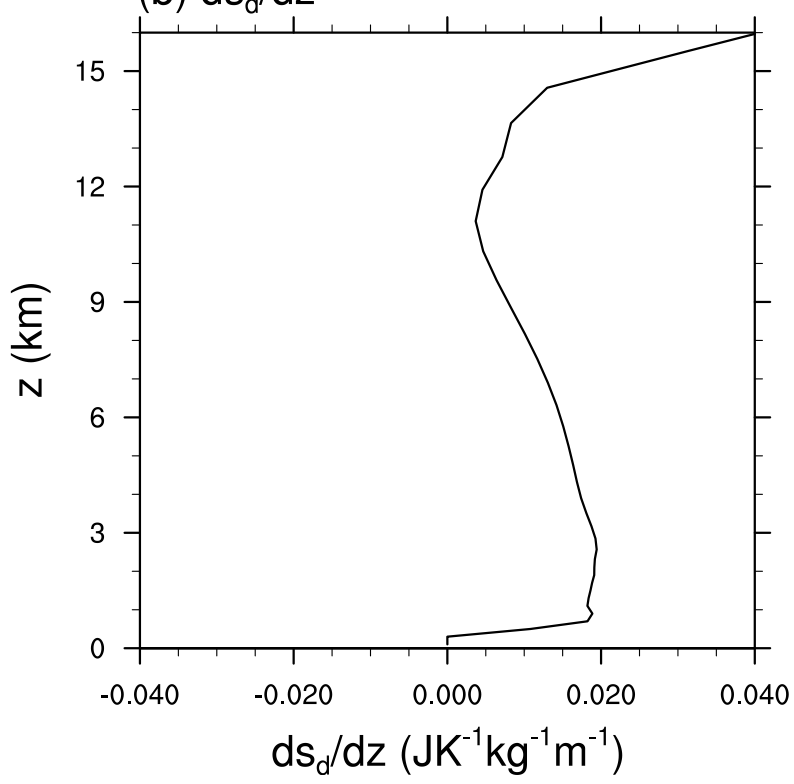

FIG. 14. (a) Vertical profiles of diagnosed subsidence velocity [Eq. (16); $\mathrm{cm} \mathrm{s}^{-1}$; blue] and azimuthal-mean actual vertical velocity ( $\mathrm{cm} \mathrm{s}^{-1}$; black) and (b) the corresponding vertical gradient of dry entropy $\left(\mathrm{J} \mathrm{K}^{-1} \mathrm{~kg}^{-1} \mathrm{~m}^{-1}\right)$ during the steady state in CTL. Values are radially averaged from 400 to $800 \mathrm{~km}$.

via removal of entropy. This indicates that both ascent and descent regions are "convective" in dry-type TCs, in contrast to CTL.

Manifested in Fig. 8, the large subsidence velocity in CTL will cause the tangential wind to decrease more rapidly in the outer region than DRY/REV according to the wind structure model of Chavas et al. (2015) and 
Chavas and Lin (2016). Given comparable outer radius of DRY/REV and CTL, the structural difference in the outer region translates to a much larger inner-core size (e.g., $r_{m}$ ) in DRY/REV relative to CTL.

\section{d. Depth of the inflow layer}

In this section, we interpret the different inflow depths in dry-type and typical TCs from a thermodynamic perspective. Evident in Fig. 3, the inflow layer reaches $4 \mathrm{~km}$ of altitude in dry-type TCs but only $1 \mathrm{~km}$ of altitude in a typical TC. Qualitatively, from a Lagrangian perspective, for an air parcel moving inward under an axisymmetric eyewall ascent, its entropy must increase according to the PI theory. As a consequence, the upper bound of the inflow layer should be limited by a height where sensible heat and water vapor diffusion fluxes can reach, given that viscous dissipation is mostly confined at the surface. In the three-dimensional TC simulated, the inward increase of azimuthal mean entropy is also contributed by vertical eddy entropy advection. The effective height of these two effects seems to be about $4-5 \mathrm{~km}$ in dry-type TCs. It seems to be further supported by the fact that even though the tropopause height increases to $15 \mathrm{~km}$ in REV from $10 \mathrm{~km}$ in DRY, the inflow depth in REV is only slightly deeper (by within $1 \mathrm{~km}$ ) than DRY. On the other hand, the inflow layer under the eyewall and under the subsidence region have the same depth in DRY and REV (Fig. 3), suggesting the importance of the processes under the subsidence region. Being just outward of the eyewall ascent, the inflow under the subsidence region should restore enough entropy to be positively buoyant so that eyewall ascent could be maintained. It turns out that the entropy restoration process is important for the inflow-layer depth.

The entropy budget under the subsidence region from 80 to $300 \mathrm{~km}$ of radius in CTL is shown in Fig. 15a. The radius range includes the intense lower- and midtropospheric low-entropy layer in Fig. 3. The most dominant feature is that the subsidence of the low-entropy air into the boundary layer induces very strong negative vertical entropy advection (Fig. 15a) at the top of boundary layer slightly below $1 \mathrm{~km}$ of altitude and this term is balanced by the convergence of water vapor diffusion flux (Fig. 15a). The horizontal advection is also negative mainly below $500 \mathrm{~m}$ of altitude because of the inward advection of the low-entropy air, which is balanced by the convergence of sensible and latent heat fluxes (Fig. 15a). The pattern suggests that subsiding air parcels have lost too much entropy during their subsidence when they reach the lower- and midtropospheric lowentropy layer (also reflected by the colored wind vector in Fig. 15a). As low as $1 \mathrm{~km}$ of altitude seems to be sufficient for them to obtain enough entropy sources
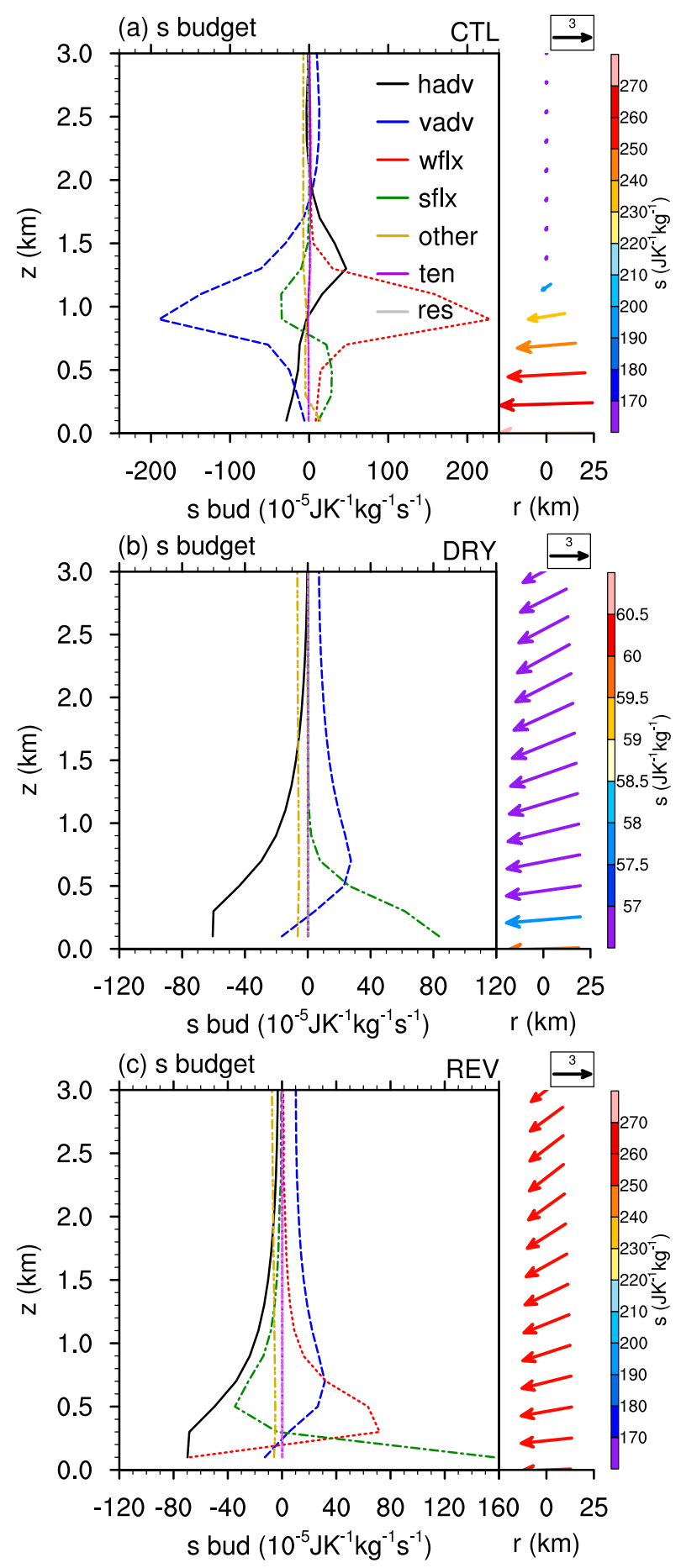

FIG. 15. (left) Azimuthally averaged entropy budgets [Eq. (7); $10^{-5} \mathrm{~J} \mathrm{~K}^{-1} \mathrm{~kg}^{-1} \mathrm{~s}^{-1}$ ] and (right) radial-vertical wind vectors $\left(\mathrm{m} \mathrm{s}^{-1}\right)$ colored by azimuthal and radial mean entropy $\left(\mathrm{J} \mathrm{K}^{-1} \mathrm{~kg}^{-1}\right)$ under the subsidence region during the steady state of (a) CTL, (b) DRY, and (c) REV. Values are radially averaged over $80-300 \mathrm{~km}$ in CTL, $700-900 \mathrm{~km}$ in DRY, and $800-1000 \mathrm{~km}$ in REV. The terms of entropy budget are $\mathrm{ADV}_{h}$ (black), $\mathrm{ADV}_{v}$ (blue), $\delta S_{\text {wflx }}$ (red), $\delta S_{\text {sflx }}$ (green), sum of other terms $\delta S_{\text {irr }}+\delta S_{\text {rad }}$ (gold), the local entropy tendency (violet), and the residual between the local tendency and the sum of all terms (gray). The reference wind vector is shown in the upper right of each panel. 
from the convergence of sensible and latent heat diffusion fluxes to restore entropy. Otherwise, their entropy would probably be unable to support the next ascent when they reach the eyewall.

In DRY and REV (Figs. 15b,c), below about $1 \mathrm{~km}$ of altitude, the dominant balance is the negative horizontal advection (Figs. 15b,c) and the entropy sources from convergence of sensible and latent heat flux (in REV only). At the lowest model level in REV, there is a net loss of water vapor due to turbulent diffusion and thus contributes negatively to the entropy budget, which is compensated by a convergence of sensible heat diffusion fluxes (Fig. 15c). The net loss of water vapor at the lowest model level is compensated by evaporation of liquid water diffused downward from above. Between 1 and $3 \mathrm{~km}$ of altitude, the dominant balance in the entropy budget is negative horizontal advection and positive vertical advection, though entropy sink from radiative cooling is not negligible (Figs. 15c,d). With entropy restoration rate of smaller magnitude and mainly near the surface, this possibly suggests that the parcels finishing subsidence do not need large entropy source to restore entropy as in a typical TC, which may allow them to turn inward at a relative higher altitude to prepare for the next ascent. This is consistent with the fact that in dry-type TCs, there is no lower- and midtropospheric low-entropy layer outside the ascending region as in CTL (colored wind vector in Figs. 15a-c). Note that the entropy in the subsidence region is equal to or even higher than the ambient environment in DRY and REV (Figs. 3a,b).

Note that the entropy flux under subsidence area (beyond the radius of $r_{\psi_{\max }}$ ) is $80 \%$ of the total in CTL, much larger than those of DRY (55\%) and REV (50\%). The larger proportion in CTL (corresponding to a larger portion of area under subsidence) is consistent with the existence of the lower troposphere low-entropy layer that suppresses convection so that eyewall cannot form easily. Finally, Figs. 15b and 15c further demonstrates an equivalent nature of DRY and REV from both wind structure and entropy budget.

\section{Conclusions and discussion}

Controlling factors for the TC structure, including its radius of the maximum wind, width of the ascending eyewall, subsidence area, and inflow-layer depth, are investigated via simulations of dry (DRY), moist reversible (REV) and typical (CTL) TCs. The main differences between dry-type and typical TCs are illustrated in Fig. 16.

First, the ER11 theory is systematically evaluated in dry-type TCs because of their wide ascents. It is found
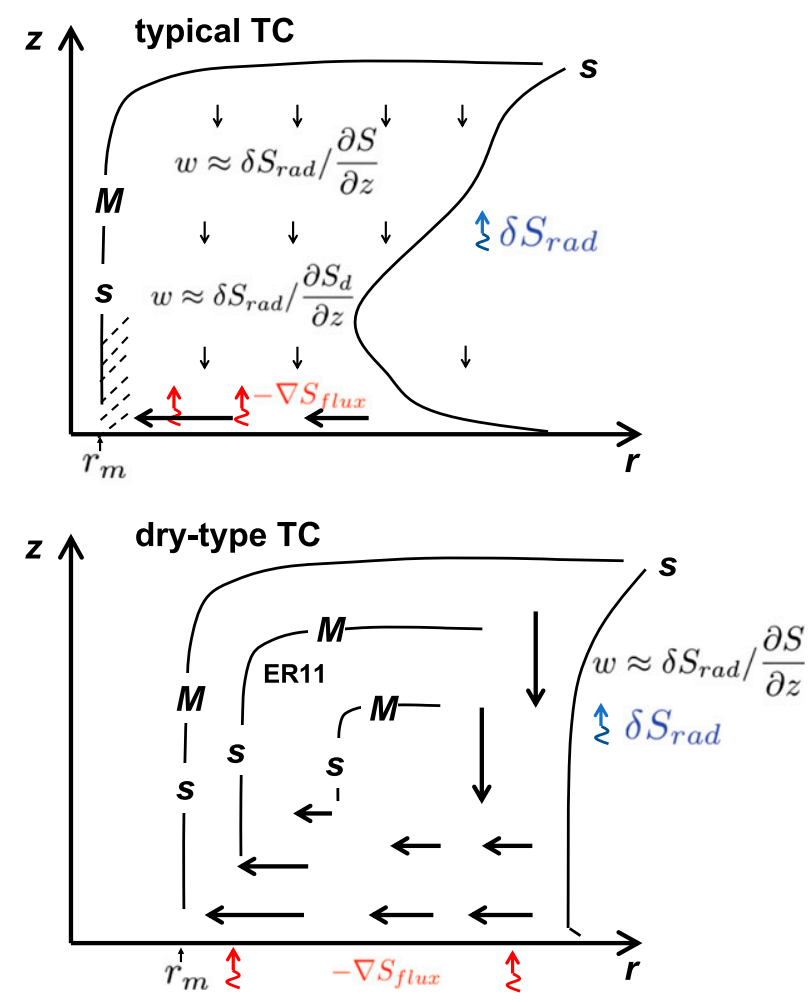

FIG. 16. Schematic plot of the main differences between (top) typical and (bottom) dry-type TCs.

that indeed, the boundary layer closure is generally valid. The numerical solution of ER11 model produces reasonable wind profiles (to a lesser extent in DRY), demonstrating the functioning of the self-stratification relation of the ER11 model. The effect of total water mixing ratio is shown to reduce the maximum intensity by $\sim 5 \mathrm{~m} \mathrm{~s}^{-1}$ at $r_{m}$. Also, the fact that steady-state intensity is independent of size proposed by ER11 is also supported in DRY. The relation between $r_{m}$ and the outer radius of ER11 is also generally valid in DRY and REV, but not in CTL. Small-scale turbulence is found active in the outflow of dry-type TCs, also supporting the concept of ER11.

Though the ER11 model depicts structures of the ascent region, the ascent width itself cannot be predicted by ER11. Compensating subsidence of eyewall ascent induces dry air outside the eyewall because of the fallout of hydrometeors in CTL. The shrink of eyewall width with the development of the mid- and uppertropospheric drying is evident at early stages. Such a drying process, known to inhibit convection by reducing buoyancy, is argued to contribute to the narrow eyewall width of CTL. Instead, such a mechanism would not work for dry-type TCs. Accompanied by the convection induced drying, $\sim 80 \%$ of the total surface entropy fluxes is contributed by surface fluxes under the subsidence 
region to restore entropy in CTL. This leads to a small area for the ascent.

With the different eyewall widths, $r_{m}$ is argued to be determined by different mechanisms in dry-type TCs and CTL. With the wide ascent, $r_{m}$ is proportional to the square of the outer radius following ER11 in dry-type TCs. With a wide subsidence in CTL, $r_{m}$ is supposed to be determined also by the outer radius via a complete model of radial wind profile by Chavas et al. (2015) and Chavas and Lin (2016).

The different subsidence velocities and inflow-layer depths in dry-type and typical TCs are attributed to a different vertical entropy gradient: an order of magnitude larger in a typical TC due to the dryness of the subsidence air parcel. The air (CTL) loses much more entropy to descend on a moist adiabat than a moist reversible air parcel (REV), which is ultimately attributed to the falling nature of hydrometeors in CTL. The orderof-magnitude greater subsidence velocity in dry-type TCs in the mid- and upper troposphere is shown to be determined by a correspondingly smaller vertical entropy gradient, given similar radiative cooling rates. On the other hand, the lower- and midtropospheric subsidence velocity in the typical moist TC is determined by the vertical gradient of dry entropy, as proposed by Emanuel (2004). The strong ascent-descent velocity asymmetry of CTL also corresponds to its much wider descent area than ascent area.

The inflow-layer depth reaches $4-5 \mathrm{~km}$ in dry-type TCs but only $1 \mathrm{~km}$ in a typical TC. The entropy restoration process limits the depth in the typical moist TC because subsidence air, with entropy much lower than the eyewall and ambient environment, must get close to the sea surface to restore enough entropy for the eyewall ascent. On the other hand, with much less entropy to be restored, the height the sensible and latent heat diffusion fluxes and vertical eddy entropy advection can effectively reach is assumed to limit the depth of inflow in dry-type TCs, which turns out to be about $4 \mathrm{~km}$ given different tropopause height for DRY and REV.

Another indication of this study is that the basic structures of real-world TC, including a narrow eyewall ascent, a small radius of maximum wind, a wide subsidence region and a shallow inflow layer, are under a constraint placed by the fallout nature of the hydrometeors, which modifies both the eyewall convective activity and the entropy structure of the subsidence region. This study also demonstrates the equivalent nature of moist reversible TC and dry TC. However, some questions, for example, what determines the outer radius in dry-type TCs and what determines the maximum streamfunction value and its radius, still remain.
TABLE 3. Comparison of intensity and size metrics of the present study and CC19. Superscript $s$ means evaluation at the lowest model level, to be consistent with $\mathrm{CC} 19 ; r_{0}^{s, t}$ is the outer radius where actual tangential wind passes $0 \mathrm{~m} \mathrm{~s}^{-1}$; and $r_{c}$ is the hypothetical outer radius of the convective core, calculated using Eq. (38) of ER11 for the present study. The value of $V_{p}$ is calculated in a similar manner as $\mathrm{CC} 19$. The values for $\mathrm{CC} 19$ are taken from their Table 1 or estimated from their Figs. 8 and 9.

\begin{tabular}{lrrrrrrr}
\hline & \multicolumn{2}{c}{ Dry } & & REV & & \multicolumn{2}{c}{ Moist } \\
\cline { 2 - 3 } & Present & CC19 & & Present & & Present & CC19 \\
\hline$v_{m}^{s}\left(\mathrm{~m} \mathrm{~s}^{-1}\right)$ & 38 & $\sim 16$ & & 41 & & 82 & $\sim 53$ \\
$V_{p}\left(\mathrm{~m} \mathrm{~s}^{-1}\right)$ & 70 & $\sim 43$ & & 78 & & 97 & $\sim 55$ \\
$V_{p} / f(\mathrm{~km})$ & 1406 & $\sim 143$ & & 1556 & & 1934 & $\sim 183$ \\
$r_{m}^{s, t}(\mathrm{~km})$ & 215 & $\sim 51$ & & 209 & & 26 & $\sim 15$ \\
$r_{0}^{s}(\mathrm{~km})$ & 887 & $\sim 226$ & & 1028 & & 863 & $\sim 476$ \\
$r_{c}(\mathrm{~km})$ & 841 & $\sim 115$ & & 857 & & 427 & $\sim 105$ \\
\hline
\end{tabular}

Additional three equivalent simulations of DRY, REV and CTL using axisymmetric configuration of CM1 are performed. The overall structures and size metrics are similar between the two groups of simulations (not shown) except that there are strong transient fluctuations in axisymmetric DRY and REV simulations due to active convective rings. Wind profile in these axisymmetric simulations, including DRY, follows the ER11 model nicely. Turbulence is more active in the outflow in axisymmetric simulations, especially for DRY and CTL. This suggests that turbulence may be important in the outflow as suggested in ER11. In general, these axisymmetric simulations appear to further suggest that dry-type TCs behave like a prototype of the ER11 model as suggested by three-dimensional simulations.

A comparison is noted between the present work and CC19 (Table 3). Consistent with CC19, the intensity of dry-type TCs are significantly smaller than $V_{p}$, whereas they are comparable in a typical moist TC (Table 3). On the other hand, the outer radius of CTL is similar to DRY/REV in the present study whereas it is about twice as large as that of the dry TC in CC19 (Table 3). With nearly constant $V_{p}$ across all simulations, CC19 showed that $V_{p} / f$ is a decent length scale of the convective-core size of TC $\left(r_{c}\right.$; Table 3$)$-thus the outer radius of the moist TC is much larger than the dry TC because of the long tail induced by the much smaller subsidence velocity in the moist TC. In the present study, with $V_{p}$ being $70 \mathrm{~m} \mathrm{~s}^{-1}, 78 \mathrm{~m} \mathrm{~s}^{-1}$, and $97 \mathrm{~m} \mathrm{~s}^{-1}$ in DRY, REV and CTL respectively, the corresponding length scale $V_{p} / f$ is over $1400 \mathrm{~km}$, more likely a scaling of the outer radius rather than the inner core size-and clearly $V_{p} / f$ does not reflect the distinction of the convective-core size between dry-type and CTL TCs $\left(r_{c}\right.$; Table 3$)$, in contrast to $\mathrm{CC} 19$. In $\mathrm{CC} 19$, they used a much larger rotation rate 
in a domain with coexisting multiple storms, which are relatively weak, turbulent, and asymmetric. These active turbulent interactions might make the dry storms smaller. Further investigation is needed to understand why $V_{p} / f$ scaling works inconsistently in the present work and CC19.

Given the equivalent nature of DRY and REV, this study also explicitly reveals that it is the subsaturation of the atmosphere matters for the substantial difference of size and structure between dry-type TCs and a typical TC, rather than the latent heat or moisture itself. This would possibly be consistent with the work of Pauluis and Held (2002a,b) and Pauluis (2011). They concluded that the mechanical efficiency (ratio of mechanical work to energy input) of a typical moist atmosphere is much smaller than that of a dry atmosphere because of irreversible entropy production by some irreversible moist processes, such as diffusion of water vapor and irreversible phase changes. The latter is mainly contributed by the evaporation of seawater into the subsaturated air at the sea surface; the irreversible evaporation of rain in subsaturated air also contributes. In REV, irreversible phase changes are eliminated because the whole atmosphere is saturated. Thus, theoretically, the mechanical efficiency of REV would be closer to DRY. Additionally, a substantial portion of the mechanical work is used to lift water in a typical atmosphere (Pauluis et al. 2000; Pauluis and Held 2002a; Pauluis and Zhang 2017), which would further reduce the amount of work to maintain the circulation. A careful analysis of energetics and how irreversible entropy production would modulate TC size and structure is left for future work.

Acknowledgments. The authors thank Dr. Chavas and two anonymous reviewers for their thoughtful and constructive review comments. This work was supported by the National Key Research Project of China (Grant 2018YFC1507001) and the National Natural Science Foundation of China (41975127).

\section{APPENDIX A}

\section{Calculation of the Theoretical Tangential Wind Profile of ER11}

Although an analytical solution of tangential wind profile is derived in Eq. (36) of ER11, it has neglected the effect of $f$. This may become inappropriate when radius becomes hundreds of kilometers, as in DRY and REV. Thus, we compute the theoretical tangential wind profile numerically from a complete model of ER11.
The thermal wind relation gives [Eq. (32) of ER11]

$$
M_{b}=r_{b}^{2}\left[\frac{1}{2} f-\left(T_{b}-T_{o}\right) \frac{d s^{*}}{d M}\right] .
$$

The quantity $d s^{*} / d M$ is prescribed by that diagnosed with Eq. (11) but without the advection and eddy flux terms on the RHS (Figs. 5a,b). The $\partial T_{o} / \partial M$ is given by the method described in section 5a. The value of $T_{b}$ is set constant for simplicity to be the value at $r_{m}$. To calculate the wind profile, we start from $r_{m}$, setting $T_{o}$ there to be the tropopause temperature of initial sounding. The value of $M$ at $r_{m}$ is computed from Eq. (A1) rather than the actual value. To march the system outward, we let $M$ increase by a small increment so that we can have a new $T_{o}$ and find an estimated $r_{b}$ through Eq. (A1). With the estimated $r_{b}$, we can find the corresponding estimated $d s^{*} / d M$. With the estimated $d s^{*} / d M$, we can calculate a new $r_{b}$ with Eq. (A1). If the difference of the new $r_{b}$ and the estimated $r_{b}$ is larger than $1 \mathrm{~m}$, then the estimated $r_{b}$ is adjusted to the difference and reiterate the above process. After determining a new $r_{b}$, we can get a new $v_{b}$ with the definition of $M$. Once $v_{b}$ is obtained, we can move to the next increment of $M$.

\section{APPENDIX B}

\section{Effect of Total Water Mixing Ratio on ER11 Model}

Although the effect of total water mixing ratio $q_{t}$ has been neglected in ER11, its effect has been considered in Emanuel (1988) and was found to reduce a TC's intensity. The basic assumption is that $q_{t}$ is a function of $M$ alone. Now that total water is actually nearly conserved in REV, it is worth checking how $q_{t}$ would modify a wind profile.

With the definition of entropy in Eq. (6), it is possible to arrive at an analogy of Eq. (10) of Emanuel (1988):

$$
\begin{aligned}
M_{b} / r_{b}^{2}= & \frac{1}{2} f-\frac{1}{1+q_{t}}\left\langle\left(T_{b}-T_{o}\right) \frac{d s^{*}}{d M}+\frac{d q_{t}}{d M}\left\{\Phi_{b}-\Phi_{o}\right.\right. \\
& +c_{l}\left[\left(T_{b}-T_{0}-T_{b} \ln \frac{T_{b}}{T_{0}}\right)\right. \\
& \left.\left.\left.-\left(T_{o}-T_{0}-T_{o} \ln \frac{T_{o}}{T_{0}}\right)\right]\right\}\right\rangle
\end{aligned}
$$

where $d q_{t} / d M$ is a function of $M, \Phi=1 / 2|\mathbf{V}|^{2}+\phi$, with $\mathbf{V}$ the total wind vector and $\phi$ the geopotential; subscript $b$ and $o$ denote evaluation at the top of the boundary layer and in the outflow, respectively. To determine $\Phi_{b}-\Phi_{o}$, we note that in inviscid steady flow with 
adiabatic transformations, $\left(1+q_{t}\right)\left(1 / 2|\mathbf{V}|^{2}+h\right)$ is constant along streamlines, with $h$ the moist static energy, so that

$$
\begin{aligned}
(1 & \left.+q_{t}\right) \Phi_{b}+\left(c_{p}+q_{t} c_{l}\right)\left(T_{b}-T_{0}\right)+L_{v b} q_{v b}^{*} \\
& =\left(1+q_{t}\right) \Phi_{o}+\left(c_{p}+q_{t} c_{l}\right)\left(T_{o}-T_{0}\right)+L_{v o} q_{v o}^{*},
\end{aligned}
$$

from which we can get $\Phi_{b}-\Phi_{o}$. The quantity $d q_{t} / d M$ is diagnosed in a similar manner as Eq. (2):

$$
\frac{d \overline{q_{t}}}{d \bar{M}} \approx-\frac{\overline{F_{q}}}{\overline{\tau_{M}}},
$$

with $F_{q}$ the surface flux of water vapor.

The computation of the wind profile with the effect of $q_{t}$ is in the same manner as in appendix A, but replace Eq. (A1) by Eq. (B1) and use Eq. (B3) for $d q_{t} / d M$. To determine $q_{v o}^{*}$ in Eq. (B2), we use an iteration method in the same manner as in section 2d of Emanuel (1986). To obtain $q_{v b}^{*}$, we also need to march pressure outward with a gradient wind balance.

\section{REFERENCES}

Bister, M., and K. A. Emanuel, 1998: Dissipative heating and hurricane intensity. Meteor. Atmos. Phys., 65, 233-240, https:// doi.org/10.1007/BF01030791.

Bjerknes, J., 1938: Saturated-adiabatic ascent of air through dryadiabatically descending environment. Quart. J. Roy. Meteor. Soc., 64, 325-330.

Bolton, D., 1980: The computation of equivalent potential temperature. Mon. Wea. Rev., 108, 1046-1053, https://doi.org/10.1175/ 1520-0493(1980)108<1046:TCOEPT>2.0.CO;2.

Bryan, G. H., and J. M. Fritsch, 2002: A benchmark simulation for moist nonhydrostatic numerical model. Mon. Wea. Rev., 130, 2917-2928, https://doi.org/10.1175/1520-0493(2002)130<2917: ABSFMN $>2.0 . \mathrm{CO} ; 2$.

the maximum intensity of tropical cyclones. J. Atmos. Sci., 66 , 3042-3060, https://doi.org/10.1175/2009JAS3038.1.

Chavas, D. R., and K. Emanuel, 2014: Equilibrium tropical cyclone size in an idealized state of axisymmetric radiative-convective equilibrium. J. Atmos. Sci., 71, 1663-1680, https://doi.org/ 10.1175/JAS-D-13-0155.1.

— tropical cyclone wind field. Part II: Wind field variability. J. Atmos. Sci., 73, 3093-3113, https://doi.org/10.1175/JAS-D-15-0185.1.

— with uniform thermal forcing: System dynamics and implications for tropical cyclone genesis and size. J. Atmos. Sci., 76, 2257-2274, https://doi.org/10.1175/JAS-D-19-0001.1.

— , N. Lin, and K. A. Emanuel, 2015: A model for the complete radial structure of the tropical cyclone wind field. Part I: Comparison with observed structure. J. Atmos. Sci., 72, 36473662, https://doi.org/10.1175/JAS-D-15-0014.1.

Črnivec, N., R. K. Smith, and G. Kilroy, 2015: Dependence of tropical cyclone intensification rate on sea surface temperature. Quart. J. Roy. Meteor. Soc., 142, 1618-1627, https://doi.org/ 10.1002/QJ.2752.
Cronin, T. W., and D. R. Chavas, 2019: Dry and semi-dry tropical cyclones. J. Atmos. Sci., 76, 2193-2212, https://doi.org/10.1175/ JAS-D-18-0357.1.

Emanuel, K. A., 1986: An air-sea interaction theory for tropical cyclones. Part I: Steady state maintenance. J. Atmos. Sci., 43, 585-605, https://doi.org/10.1175/1520-0469(1986)043<0585: AASITF $>2.0 . \mathrm{CO} ; 2$.

, 1988: The maximum intensity of hurricanes. J. Atmos. Sci., $\mathbf{4 5}$, 1143-1155, https://doi.org/10.1175/1520-0469(1988)045<1143: $\mathrm{TMIOH}>2.0 . \mathrm{CO} ; 2$.

- 1991: The theory of hurricanes. Annu. Rev. Fluid Mech., 23, 179-196, https://doi.org/10.1146/annurev.fl.23.010191.001143.

_- 1994: Atmospheric Convection. Oxford University Press, 580 pp.

- 2004: Tropical cyclone energetics and structure. Atmospheric Turbulence and Mesoscale Meteorology, E. Fedorovich, R. Rotunno, and B. Stevens, Eds., Cambridge University Press, 165-192.

_ 2012: Self-stratification of tropical cyclone outflow. Part II: Implications for storm intensification. J. Atmos. Sci., 69, 988996, https://doi.org/10.1175/JAS-D-11-0177.1.

_ outflow. Part I: Implications for storm structure. J. Atmos. Sci., 68, 2236-2249, https://doi.org/10.1175/JAS-D-10-05024.1.

Freismuth, T. M., B. Rutherford, M. A. Boothe, and M. T. Montgomery, 2016: Why did the storm ex-Gaston (2010) fail to redevelop during the PREDICT experiment? Atmos. Chem. Phys., 16, 8511-8519, https://doi.org/10.5194/acp-168511-2016.

James, R. P., and P. M. Markowski, 2010: A numerical investigation of the effects of dry air aloft on deep convection. Mon. Wea. Rev., 138, 140-161, https://doi.org/10.1175/ 2009MWR3018.1.

Kessler, E., 1995: On the continuity and distribution of water substance in atmospheric circulations. Atmos. Res., 38, 109145, https://doi.org/10.1016/0169-8095(94)00090-Z.

Kilroy, G., and R. K. Smith, 2012: A numerical study of rotating convection during tropical cyclogenesis. Quart. J. Roy. Meteor. Soc., 139, 1255-1269, https://doi.org/10.1002/qj.2022.

Montgomery, M. T., J. Persing, and R. K. Smith, 2019: On the hypothesized outflow control of tropical cyclone intensification. Quart. J. Roy. Meteor. Soc., 145, 1309-1322, https:// doi.org/10.1002/qj.3479.

Mrowiec, A., S. T. Garner, and O. M. Pauluis, 2011: Axisymmetric hurricane in a dry atmosphere: Theoretical framework and numerical experiments. J. Atmos. Sci., 68, 1607-1619, https:// doi.org/10.1175/2011JAS3639.1.

Pauluis, O. M., 2011: Water vapor and mechanical work: A comparison of Carnot and steam cycles. J. Atmos. Sci., 68, 91-102, https://doi.org/10.1175/2010JAS3530.1.

— radiative-convective equilibrium. Part I: Maximum work and frictional dissipation. J. Atmos. Sci., 59, 125-139, https://doi.org/ 10.1175/1520-0469(2002)059<0125:EBOAAI >2.0.CO;2.

, and - 2002b: Entropy budget of an atmosphere in radiative-convective equilibrium. Part II: Latent heat transport and moist processes. J. Atmos. Sci., 59, 140-149, https://doi.org/ 10.1175/1520-0469(2002)059<0140:EBOAAI>2.0.CO;2.

—, and F. Zhang, 2017: Reconstruction of thermodynamic cycles in a high-resolution simulation of a hurricane. J. Atmos. Sci., 74, 3367-3381, https://doi.org/10.1175/JAS-D-16-0353.1.

__, V. Balaji, and I. M. Held, 2000: Frictional dissipation in a precipitating atmosphere. J. Atmos. Sci., 57, 989-994, 
https://doi.org/10.1175/1520-0469(2000)057<0989:FDIAPA > 2.0.CO;2.

Peng, K., R. Rotunno, and G. H. Bryan, 2018: Evaluation of a timedependent model for the intensification of tropical cyclones. J. Atmos. Sci., 75, 2125-2138, https://doi.org/10.1175/JAS-D17-0382.1.

Persing, J., M. T. Montgomery, J. C. McWilliams, and R. K. Smith, 2013: Asymmetric and axisymmetric dynamics of tropical cyclones. Atmos. Chem. Phys., 13, 12 299-12 341, https://doi.org/ 10.5194/acp-13-12299-2013.

Rhines, P. B., 1975: Waves and turbulence on a beta-plane. J. Fluid Mech., 69, 417-443, https://doi.org/10.1017/S0022112075001504.

Romps, D. M., 2008: The dry-entropy budget of a moist atmosphere. J. Atmos. Sci., 65, 3779-3799, https://doi.org/10.1175/ 2008JAS2679.1.
Rotunno, R., and K. A. Emanuel, 1987: An air-sea interaction theory for tropical cyclones. Part II: Evolutionary study using a nonhydrostatic axisymmetric numerical model. J. Atmos. Sci., 43, 542-561, https://doi.org/10.1175/1520-0469(1987) $044<0542$ :AAITFT $>2.0$.CO;2.

Smith, R. K., and M. T. Montgomery, 2012: Observations of the convective environment in developing and non-developing tropical disturbances. Quart. J. Roy. Meteor. Soc., 138, 17211739, https://doi.org/10.1002/qj.1910.

Tao, D., K. A. Emanuel, F. Zhang, R. Rotunno, M. M. Bell, and R. G. Nystrom, 2019: Evaluation of the assumptions in the steady-state tropical cyclone self-stratified outflow using three-dimensional convection-allowing simulations. J. Atmos. Sci., 76, 2995-3009, https://doi.org/10.1175/JASD-19-0033.1. 